\title{
Genetic Variants of Methyl Metabolizing Enzymes and Epigenetic Regulators: Associations with Promoter CpG Island Hypermethylation in Colorectal Cancer
}

Citation for published version (APA):

de Vogel, S. C., Wouters, K. A., Gottschalk, R. W., van Schooten, F. J., de Goeij, A. F., de Bruine, A. P., Goldbohm, R. A., van den Brandt, P. A., Weijenberg, M. P., \& van Engeland, M. (2009). Genetic Variants of Methyl Metabolizing Enzymes and Epigenetic Regulators: Associations with Promoter CpG Island Hypermethylation in Colorectal Cancer. Cancer Epidemiology Biomarkers \& Prevention, 18(11), 30863096. https://doi.org/10.1158/1055-9965.EPI-09-0289

Document status and date:

Published: 01/01/2009

DOI:

10.1158/1055-9965.EPI-09-0289

Document Version:

Publisher's PDF, also known as Version of record

Document license:

Taverne

Please check the document version of this publication:

- A submitted manuscript is the version of the article upon submission and before peer-review. There can be important differences between the submitted version and the official published version of record.

People interested in the research are advised to contact the author for the final version of the publication, or visit the DOI to the publisher's website.

- The final author version and the galley proof are versions of the publication after peer review.

- The final published version features the final layout of the paper including the volume, issue and page numbers.

Link to publication

\footnotetext{
General rights rights.

- You may freely distribute the URL identifying the publication in the public portal. please follow below link for the End User Agreement:

www.umlib.nl/taverne-license

Take down policy

If you believe that this document breaches copyright please contact us at:

repository@maastrichtuniversity.nl

providing details and we will investigate your claim.
}

Copyright and moral rights for the publications made accessible in the public portal are retained by the authors and/or other copyright owners and it is a condition of accessing publications that users recognise and abide by the legal requirements associated with these

- Users may download and print one copy of any publication from the public portal for the purpose of private study or research.

- You may not further distribute the material or use it for any profit-making activity or commercial gain

If the publication is distributed under the terms of Article $25 \mathrm{fa}$ of the Dutch Copyright Act, indicated by the "Taverne" license above, 


\title{
Genetic Variants of Methyl Metabolizing Enzymes and Epigenetic Regulators: Associations with Promoter CpG Island Hypermethylation in Colorectal Cancer
}

\author{
Stefan de Vogel, ${ }^{1,2}$ Kim A.D. Wouters, ${ }^{2}$ Ralph W.H. Gottschalk, ${ }^{3}$ Frederik J. van Schooten, ${ }^{3}$ \\ Anton F.P.M. de Goeij, ${ }^{2}$ Adriaan P. de Bruïne, ${ }^{2}$ Royle A. Goldbohm, \\ Piet A. van den Brandt, ${ }^{1}$ Matty P. Weijenberg, ${ }^{1}$ and Manon van Engeland ${ }^{2}$ \\ Departments of ${ }^{1}$ Epidemiology and ${ }^{2}$ Pathology, GROW-School for Oncology and Developmental Biology; ${ }^{3}$ Department of Health \\ Risk Analysis and Toxicology, Nutrition and Toxicology Research Institute Maastricht, Maastricht University, Maastricht, \\ the Netherlands; and ${ }^{4}$ Department of Prevention and Health, TNO Quality of Life, Leiden, the Netherlands
}

\begin{abstract}
Aberrant DNA methylation affects carcinogenesis of colorectal cancer. Folate metabolizing enzymes may influence the bioavailability of methyl groups, whereas DNA and histone methyltransferases are involved in epigenetic regulation of gene expression. We studied associations of genetic variants of folate metabolizing enzymes (MTHFR, MTR, and MTRR), DNA methyltransferase $D N M T 3 b$, and histone methyltransferases (EHMT1, EHMT2, and PRDM2), with colorectal cancers, with or without the $\mathrm{CpG}$ island methylator phenotype (CIMP), MLH1 hypermethylation, or microsatellite instability. Incidence rate ratios were calculated in case-cohort analyses, with common homozygotes as reference, among 659 cases and 1,736 subcohort members of the Netherlands Cohort Study on diet and cancer $(n=120,852)$. Men with the MTHFR $677 T T$ genotype were at decreased colorectal cancer risk (incidence rate ratio, $0.49 ; P=0.01$ ), but the $T$ allele was associated with increased risk in women (incidence rate ratio, $1.39 ; P=0.02)$. The $M T R$ 2756 G genotype was associated with increased

colorectal cancer risk (incidence rate ratio, 1.58; $P=$ 0.04), and inverse associations were observed among women carrying $D N M T 3 b C \rightarrow T$ (rs406193; incidence rate ratio, $0.72 ; P=0.04$ ) or $E H M T 2 G \rightarrow A$ (rs535586; incidence rate ratio, $0.76 ; P=0.05)$ polymorphisms. Although significantly correlated $(P<0.001)$, only $41.5 \%$ and $33.3 \%$ of CIMP tumors harbored $M L H 1$ hypermethylation or microsatellite instability, respectively. We observed inverse associations between MTR A2756G and CIMP among men (incidence rate ratio, 0.58; $P=0.04$ ), and between MTRR A66G and MLH1 hypermethylation among women (incidence rate ratio, $0.55 ; P=0.02)$. In conclusion, MTHFR, MTR, $D N M T 3 b$, and EHMT2 polymorphisms are associated with colorectal cancer, and rare variants of MTR and $M T R R$ may reduce promoter hypermethylation. The incomplete overlap between CIMP, MLH1 hypermethylation, and microsatellite instability indicates that these related "methylation phenotypes" may not be similar and should be investigated separately. (Cancer Epidemiol Biomarkers Prev 2009;18(11):3086-96)
\end{abstract}

\section{Introduction}

Aberrant DNA methylation is an important epigenetic modification that may affect cancer susceptibility (1). Folate metabolizing enzymes are involved in the provision of methyl groups, and the bioavailability of methyl groups is dependent on the activity of these enzymes. Singlenucleotide polymorphisms in these genes have been reported to alter enzymatic activity. For example, rare variants of the $C 677 T$ and $A 1298 C$ polymorphisms in the methylene tetrahydrofolate reductase (MTHFR) gene result in reduced enzymatic activity $(2,3)$ and were inversely associated with colorectal cancer in several

\footnotetext{
Received 3/30/09; revised 7/14/09; accepted 9/8/09; published OnlineFirst 10/20/09. Grant support: The Dutch Cancer Society (UM2004-3171 and UM99-1980).

Note: Supplementary data for this article are available at Cancer Epidemiology, Biomarkers and Prevention Online (http://cebp.aacrjournals.org/).

Requests for reprints: Manon van Engeland, Department of Pathology, GROW-School for Oncology and Developmental Biology, Maastricht University, P.O. Box 616, 6200MD Maastricht, the Netherlands. Phone: 31-43-3874622; Fax: 31-43-3876613.

E-mail: m.vanengeland@path.unimaas.nl

Copyright (C) 2009 American Association for Cancer Research.

doi:10.1158/1055-9965.EPI-09-0289
}

observational studies $(4,5)$. However, it was suggested that subjects having the MTHFR 677TT or 1298CC genotypes were more likely to develop colorectal cancer showing a CpG island hypermethylation phenotype $(6,7)$ or microsatellite instability (8). Genetic variants of methionine synthase (MTR) have been studied less extensively, but the rare GG variant of the MTR A2756G single-nucleotide polymorphism was suggested to decrease colorectal cancer risk in two studies $(9,10)$. In contrast, it was suggested that some of the rare genetic variants of methionine synthase reductase (MTRR) may be associated with an increased risk for colorectal adenomas and carcinomas (11-13).

Whereas the aforementioned one carbon-metabolizing enzymes are involved in the provision of methyl groups, DNA methyltransferases catalyze the transfer of these methyl groups from S-adenosylmethionine into CPG dinucleotides of DNA. The DNA methyltransferase $3 b$ (DNMT3b) is involved in de novo methylation (14) and may influence epigenetic regulation of gene expression and cancer cell growth. In this respect, experimental research suggested that DNMT3b depletion can reduce aberrant promoter $\mathrm{CPG}$ island hypermethylation in 


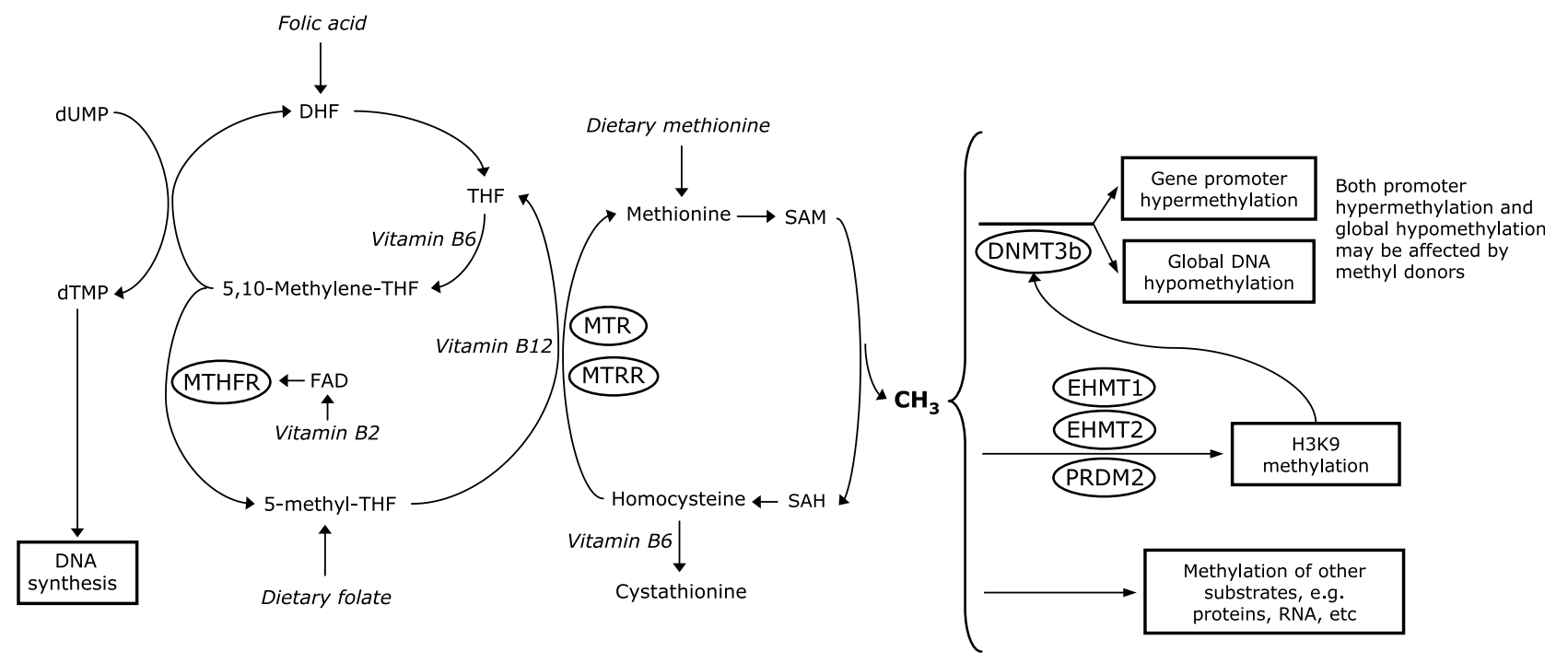

Balance towards increased bioavailability of methyl groups and thereby a potential effect on DNA methylation

Figure 1. Folate metabolizing enzymes, DNA methyltransferases, histone methyl transferases, and DNA methylation. Potential targets of methyl groups are DNA, lysines (among which is histone H3 Lys-9), or other substrates, for example, proteins, RNA, etc. DNMT3b activity and promoter CpG island hypermethylation may depend on previous methylation of histone H3 Lys-9. Ovals represent the enzymes, of which single-nucleotide polymorphisms are investigated in this study. dUMP, deoxyuridine monophosphate; dTMP, deoxythymidine monophosphate; DHF, dihydrofolate; THF, tetrahydrofolate; FAD, flavine adenine dinucleotide; SAM, S-adenosyl methionine; SAH, S-adenosyl homocysteine; MTHFR, methylene tetrahydrofolate reductase; MTR, methionine synthase; MTRR, methionine synthase reductase; DNMT3b, DNA methyltransferase 3b; PRDM2, PR domain 2; EHMT, euchromatine histone methyltransferase.

cancer cells (15-17), whereas DNMT3b overexpression initiated promoter hypermethylation of tumor suppressor genes and the formation of colonic miroadenomas (18). In addition, it was observed that expression of DNMT3b was associated with $p 16$ and RASSF1A promoter methylation in non-small cell lung cancer (19) and with a promoter hypermethylator phenotype in breast cancer (20). Several single-nucleotide polymorphisms of the DNMT3b gene have been identified, which may affect catalytic activity of the DNMT3b enzyme. For example, the rare $T$ allele of the DNMT3b $C \rightarrow T$ (rs2424913) polymorphism was found to significantly increase DNTM $3 b$ promoter activity and was associated with an increased risk for lung cancer (21), prostate cancer (22), and colorectal polyps, including colorectal adenomas (23), or with prognosis of head and neck cancer (24). Moreover, subjects with hereditary nonpolyposis colorectal cancer carrying the TT genotype developed colorectal cancer at a younger age compared with those homozygous for the wild-type DNMT3b CC allele (25). However, the association between the DNMT3b $C \rightarrow T$ (rs2424913) polymorphism and colorectal cancer risk has not previously been investigated.

It has been hypothesized that DNA methyltransferases may only have an effect on the chromatin if histone $\mathrm{H} 3$ Lys-9 is first methylated by histone methyltransferases (Fig. 1) and that DNA methylation may thus depend on the activity of histone methyltransferases (26). Experimental research also indicated that methylation of histone H3 Lys-9 and other histones play a critical role in maintaining epigenetic silencing by promoter hypermethylation of genes involved in colorectal cancer (27). The retinoblastoma protein interacting zinc finger gene (RIZ or PRDM2) is a histone methyltransferase that may act as a tumor suppressor, and PRDM2 frameshift mutations have been observed in colorectal cancers showing microsatellite instability (28). Genetic variation of PRDM2 may be hypothesized to affect its gene activity, and single-nucleotide polymorphisms in PRDM2 were observed to be inversely associated with lung cancer (29). However, an increased risk for breast cancer was suggested (30). Other genes that have been identified as histone methyltransferases are euchromatin histone methyltransferase-1 (EHMT1) and -2 (EHMT2), and genetic variants of these genes were modestly associated with breast cancer risk in a large case-control study (30). However, the potential impact of genetic variants of PRDM2, EHMT1, and EHMT2 has not previously been studied in relation to colorectal cancer.

The aim of this study was to determine the occurrence of single-nucleotide polymorphisms in genes encoding folate metabolizing enzymes (MTHFR, MTR, and MTRR), the DNA methyltransferase DNMT3b, and histone methyltransferases (EHMT1, EHMT2, and PRDM2) in a 
large population-based prospective cohort study in the Netherlands. We estimated associations of these singlenucleotide polymorphisms with overall colorectal cancer risk and with colorectal cancers with or without the $\mathrm{CpG}$ island methylator phenotype (CIMP), MLH1 hypermethylation, or microsatellite instability.

\section{Subjects and Methods}

Study Population and Tumor Tissue Samples. Tumor material was obtained from incident colorectal cancer patients from the Netherlands cohort study, which has been described in detail elsewhere (31). Briefly, this prospective cohort study was initiated in September 1986 and includes 58,279 men and 62,573 women with the age of 55 to $69 \mathrm{y}$ and free of disease at baseline. The cohort is followed for cancer occurrence by annual record linkage to the Netherlands Cancer Registry and to the Pathologisch Anatomisch Landelijk Geautomatiseerd Archief, a nationwide network and registry of histopathology and cytopathology reports (32, 33). At baseline, participants filled out a self-administered food frequency questionnaire, by which information was obtained about age, sex, family history of colorectal cancer, smoking behavior, and body mass index. A subcohort of 5,000 subjects was randomly selected after baseline exposure measurement to estimate accumulation of person-time in the cohort through biennial follow-up of vital status. Cases with prevalent cancer other than nonmelanoma skin cancer were excluded from this subcohort, which left 4,774 men and women eligible for analysis. Tumor material of the colorectal cancer patients was collected after approval by the ethical review boards of Maastricht University, the Netherlands Cancer Registry, and the Pathologisch Anatomisch Landelijk Geautomatiseerd Archief. During a follow-up period of $7.3 \mathrm{y}$ after baseline, 734 incident colorectal cancer patients were identified who had an available Pathologisch Anatomisch Landelijk Geautomatiseerd Archief report of the lesion, as well as a sufficient amount of isolated DNA.

Collection of Mouth Swabs of Subcohort Members. Subcohort members still alive in December $2000(n=$ 3,579 ) were contacted and asked to collect mouth swabs. Four cotton swabs in a small nonwoven polyethylene envelope were mailed to each subject, including a simple protocol on how to use them. After receipt, the swabs were placed in a falcon tube containing $2.0 \mathrm{~mL}$ buffer solution (100 mmol/L NaCl; $10 \mathrm{mmol} / \mathrm{L} \mathrm{EDTA;} 10 \mathrm{mmol} / \mathrm{L}$ Tris, $\mathrm{pH} 8$; with $0.2 \mathrm{mg} / \mathrm{mL}$ proteinase $\mathrm{K}$ and $0.5 \% \mathrm{w} / \mathrm{v}$ SDS). The lysed solution was kept stored at room temperature in the dark. In total, 1,929 subcohort members (54\%) returned the mouth swabs with informed consent. The average DNA yield per cotton swab was 0.1 to $10 \mu \mathrm{g}$, which corresponded with data from literature (34). DNA could successfully be isolated from 1,829 subcohort members who also had complete follow-up information.

Genotyping Analyses. Nine fragments containing the MTHFR, MTR, MTRR, DNMT3b, EHMT1, EHMT2, and $P R D M 2$ single-nucleotide polymorphisms were amplified using multiplex PCR amplification and single-base extension reactions, as described previously by Knaapen et al. (35). Genomic DNA (50 ng) was added to $1 \times$ PCR buffer (Invitrogen), $1.75 \mathrm{mmol} / \mathrm{L} \mathrm{MgCl}_{2}$ (Invitrogen), $0.4 \mu \mathrm{mol} / \mathrm{L} \mathrm{dNTPs}$ (Amersham Bioscience), $100 \mathrm{nmol} / \mathrm{L}$ of each primer (Eurogentec), and 0.25 U Platinum Taq (Invitrogen) in a final volume of $10 \mu \mathrm{L}$. PCR conditions were as follows: $3 \mathrm{~min}$ at $94^{\circ} \mathrm{C}, 35$ cycles of $30 \mathrm{sec}$ at $94^{\circ} \mathrm{C}, 30 \mathrm{sec}$ at $62^{\circ} \mathrm{C}$ and $30 \mathrm{sec}$ at $72^{\circ} \mathrm{C}$, and a final extension for $5 \mathrm{~min}$ at $72^{\circ} \mathrm{C}$ and $4 \mathrm{~min}$ at $20^{\circ} \mathrm{C}$. To degrade excess PCR primers and dNTPs, $5 \mu \mathrm{L}$ multiplex PCR product was incubated with $2 \mu \mathrm{L}$ EXO-SAP IT (Amersham Bioscience) at $37^{\circ} \mathrm{C}$ for $15 \mathrm{~min}$, followed by $80^{\circ} \mathrm{C}$ for $15 \mathrm{~min}$ to deactivate the enzyme. The multiplex single-base extension reaction was done using a SNaPShot multiplex kit, as described by the manufacturer (Applied Biosystems). single-base extension primers were designed to bind immediately adjacent $5^{\prime}$ to the Single-nucleotide polymorphism of interest with a template-specific part of 20 to $33 \mathrm{bp}$ and a temperature of $60^{\circ} \mathrm{C}$. rs numbers of the single-nucleotide polymorphisms and primer sequences are shown in Supplementary Table S1. During thermal cycling, the primers are extended at their $3^{\prime}$ end with a single dideoxyribonucleoside triphosphate labeled with a distinct fluorophore, revealing the genotype of the single-nucleotide polymorphism. Single-base extension was done using 25 cycles of $96^{\circ} \mathrm{C}$ for $10 \mathrm{~s}$ and $60^{\circ} \mathrm{C}$ for $30 \mathrm{~s}$. Following cycling, the reaction was treated with 1 U Shrimp Alkaline Phosphatase (Amersham Bioscience) at $37^{\circ} \mathrm{C}$ for $1 \mathrm{~h}$ to degrade the unincorporated dideoxynucleotide triphosphates, followed by enzyme deactivation at $75^{\circ} \mathrm{C}$ for $15 \mathrm{~min}$. One microliter of single-base extension product was mixed with $13 \mu \mathrm{L}$ of $\mathrm{Hi}$-Di formamide (Applied Biosystems) and $0.4 \mu \mathrm{L}$ of Genescan- 120 size standard (Applied Biosystems), subsequently denatured at $95^{\circ} \mathrm{C}$ for $5 \mathrm{~min}$, and then analyzed on an ABI Prism 3100 genetic analyzer using Genemapper Analysis software (version 4.0).

To validate the genotype data, we sequenced every fragment containing a specific single-nucleotide polymorphism in a subset of 30 samples, including 10 colorectal cancer cases, 10 female, and 10 male subcohort members with mouth swabs. Sequencing was done using the BigDye Terminater v1.1 cycle sequencing kit, following the manufacturer's recommendations using the ABI 3700 genetic analyzer. The sequencing results were similar to the $\mathrm{SNaPShot} \mathrm{results} \mathrm{for} \mathrm{all} \mathrm{but} \mathrm{one}(99.6 \%)$ of the nine single-nucleotide polymorphisms within these 30 samples. Reproducibility of the SNaPShot analysis was established by subjecting 93 samples, composed of 31 colorectal cancer cases, 31 female, and 31 male subcohort members with mouth swabs, twice to the complete SNaPShot analysis procedure, from multiplex PCR of genomic DNA to the genetic analysis of the samples. We observed that the analyses could be reproduced in $99.5 \%$ of these cases.

Promoter Methylation Analyses. The CIMP was defined by promoter hypermethylation of at least three of five methylation markers (CACNA1G, IGF2, NEUROG1, RUNX3, and SOCS1), as suggested by Weisenberger et al. (36). DNA methylation in the CpG islands of these five CIMP markers and of the MLH1 gene was determined by Methylation Specific PCR (37). Bisulfite modification was carried out on $500 \mathrm{ng}$ DNA using a commercially available kit (Zymo Research). To facilitate Methylation Specific PCR analysis on DNA retrieved from formalin-fixed, paraffinembedded tissue, DNA was first amplified with flanking PCR primers that amplify bisulfite modified DNA but do not preferentially amplify methylated or unmethylated DNA. The resulting fragment was used as a template for 
Table 1. Prevalence of genotypes of subcohort members and colorectal cancer patients in the Netherlands cohort study

\begin{tabular}{|c|c|c|c|c|c|c|c|c|}
\hline \multirow[t]{2}{*}{ Gene and SNP } & \multirow{2}{*}{$\begin{array}{l}\text { dbSNP } \\
\text { number }\end{array}$} & \multirow[t]{2}{*}{ Genotype (MAF) } & \multicolumn{2}{|c|}{ Men and women } & \multicolumn{2}{|c|}{ Men } & \multicolumn{2}{|c|}{ Women } \\
\hline & & & $\begin{array}{l}\text { Subcohort } \\
(\mathrm{HWE})^{*}\end{array}$ & CRC cases & $\begin{array}{l}\text { Subcohort } \\
(\mathrm{HWE})^{*}\end{array}$ & CRC cases & $\begin{array}{l}\text { Subcohort } \\
(\mathrm{HWE})^{*}\end{array}$ & CRC cases \\
\hline $\begin{array}{r}\text { MTHFR } \\
\text { C677T }\end{array}$ & rs1801133 & $\begin{array}{c}C C \\
C T \\
T T \\
(0.30)\end{array}$ & $\begin{array}{c}876(48.9 \%) \\
750(41.8 \%) \\
167(9.3 \%) \\
(0.72)\end{array}$ & $\begin{array}{c}318(46.2 \%) \\
320(46.4 \%) \\
51(7.4 \%)\end{array}$ & $\begin{array}{c}409(45.4 \%) \\
405(44.9 \%) \\
87(9.7 \%) \\
(0.36)\end{array}$ & $\begin{array}{c}179(46.8 \%) \\
184(48.2 \%) \\
19(5.0 \%)\end{array}$ & $\begin{array}{c}467(52.3 \%) \\
345(38.7 \%) \\
80(9.0 \%) \\
(0.16)\end{array}$ & $\begin{array}{r}139(45.3 \%) \\
136(44.3 \%) \\
32(10.4 \%)\end{array}$ \\
\hline $\begin{array}{l}\text { MTHFR } \\
\text { A1298C }\end{array}$ & rs1801131 & $\begin{array}{l}A A \\
A C \\
C C \\
(0.37)\end{array}$ & $\begin{array}{c}735(41.6 \%) \\
774(43.8 \%) \\
258(14.6 \%) \\
(0.02)\end{array}$ & $\begin{array}{l}299(43.7 \%) \\
275(40.2 \%) \\
110(16.1 \%)\end{array}$ & $\begin{array}{c}345(39.3 \%) \\
423(48.2 \%) \\
110(12.5 \%) \\
(0.26)\end{array}$ & $\begin{array}{r}167(43.8 \%) \\
166(43.6 \%) \\
48(12.6 \%)\end{array}$ & $\begin{array}{c}390(43.9 \%) \\
351(39.5 \%) \\
148(16.6 \%) \\
(<0.001)\end{array}$ & $\begin{array}{r}132(43.6 \%) \\
109(36.0 \%) \\
62(20.4 \%)\end{array}$ \\
\hline MTR A2756G & rs1805087 & $\begin{array}{c}A A \\
A G \\
G G \\
(0.19)\end{array}$ & $\begin{array}{c}1190(65.9 \%) \\
543(30.1 \%) \\
72(4.0 \%) \\
(0.31)\end{array}$ & $\begin{array}{c}449(64.5 \%) \\
204(29.3 \%) \\
43(6.2 \%)\end{array}$ & $\begin{array}{c}589(65.1 \%) \\
281(31.0 \%) \\
35(3.9 \%) \\
(0.84)\end{array}$ & $\begin{array}{c}257(66.4 \%) \\
108(27.9 \%) \\
22(5.7 \%)\end{array}$ & $\begin{array}{c}601(66.8 \%) \\
262(29.1 \%) \\
37(4.1 \%) \\
(0.22)\end{array}$ & $\begin{array}{c}192(62.1 \%) \\
96(31.1 \%) \\
21(6.8 \%)\end{array}$ \\
\hline MTRR A66G & rs1801394 & $\begin{array}{l}A A \\
A G \\
G G \\
(0.56)\end{array}$ & $\begin{array}{c}367(20.4 \%) \\
833(46.4 \%) \\
597(33.2 \%) \\
(0.01)\end{array}$ & $\begin{array}{l}136(19.5 \%) \\
338(48.3 \%) \\
225(32.2 \%)\end{array}$ & $\begin{array}{c}193(21.4 \%) \\
399(44.2 \%) \\
310(34.4 \%) \\
(0.003)\end{array}$ & $\begin{array}{r}65(16.7 \%) \\
196(50.2 \%) \\
129(33.1 \%)\end{array}$ & $\begin{array}{c}174(19.4 \%) \\
434(48.5 \%) \\
287(32.1 \%) \\
(0.67)\end{array}$ & $\begin{array}{r}71(23.0 \%) \\
142(45.9 \%) \\
96(31.1 \%)\end{array}$ \\
\hline$\underset{\mathrm{CNMT} 3 b}{\operatorname{CN} T^{\ddagger}}$ & rs2424913 & $\begin{array}{c}C C \\
C T \\
T T \\
(0.42)\end{array}$ & $\begin{array}{c}597(32.9 \%) \\
895(49.5 \%) \\
318(17.6 \%) \\
(0.58)\end{array}$ & $\begin{array}{l}240(34.1 \%) \\
348(49.5 \%) \\
115(16.4 \%)\end{array}$ & $\begin{array}{c}303(33.4 \%) \\
449(49.6 \%) \\
154(17.0 \%) \\
(0.57)\end{array}$ & $\begin{array}{r}132(33.9 \%) \\
184(47.3 \%) \\
73(18.8 \%)\end{array}$ & $\begin{array}{c}294(32.5 \%) \\
446(49.4 \%) \\
164(18.2 \%) \\
(0.82)\end{array}$ & $\begin{array}{r}108(34.4 \%) \\
164(52.2 \%) \\
42(13.4 \%)\end{array}$ \\
\hline $\begin{array}{c}\mathrm{DNMT} 3 b \\
\mathrm{C} \rightarrow T^{\ddagger}\end{array}$ & rs406193 & $\begin{array}{c}C C \\
C T \\
T T \\
(0.14)\end{array}$ & $\begin{array}{c}1331(74.3 \%) \\
415(23.1 \%) \\
46(2.6 \%) \\
(0.05)\end{array}$ & $\begin{array}{c}528(75.6 \%) \\
152(21.8 \%) \\
18(2.6 \%)\end{array}$ & $\begin{array}{c}686(76.7 \%) \\
190(21.2 \%) \\
19(2.1 \%) \\
(0.18)\end{array}$ & $\begin{array}{c}291(74.6 \%) \\
88(22.6 \%) \\
11(2.8 \%)\end{array}$ & $\begin{array}{c}645(71.9 \%) \\
225(25.1 \%) \\
27(3.0 \%) \\
(0.18)\end{array}$ & $\begin{array}{c}237(76.9 \%) \\
64(20.8 \%) \\
7(2.3 \%)\end{array}$ \\
\hline $\begin{array}{l}E H M T 1 \\
\quad G \rightarrow A^{\ddagger}\end{array}$ & rs4634736 & $\begin{array}{c}G G \\
G A \\
A A \\
(0.10)\end{array}$ & $\begin{array}{c}1444(80.6 \%) \\
334(18.6 \%) \\
14(0.8 \%) \\
(0.27)\end{array}$ & $\begin{array}{c}568(81.7 \%) \\
121(17.4 \%) \\
6(0.9 \%)\end{array}$ & $\begin{array}{c}730(81.4 \%) \\
163(18.2 \%) \\
4(0.4 \%) \\
(0.11)\end{array}$ & $\begin{array}{c}320(83.1 \%) \\
64(16.6 \%) \\
1(0.3 \%)\end{array}$ & $\begin{array}{c}714(79.8 \%) \\
171(19.1 \%) \\
10(1.1 \%) \\
(0.95)\end{array}$ & $\begin{array}{c}248(80.0 \%) \\
57(18.4 \%) \\
5(1.6 \%)\end{array}$ \\
\hline $\begin{array}{r}E H M T 2 \\
G \rightarrow A\end{array}$ & rs535586 & $\begin{array}{c}G G \\
G A \\
A A \\
(0.35)\end{array}$ & $\begin{array}{c}755(42.2 \%) \\
810(45.3 \%) \\
223(12.5 \%) \\
(0.80)\end{array}$ & $\begin{array}{r}297(42.9 \%) \\
311(44.9 \%) \\
84(12.2 \%)\end{array}$ & $\begin{array}{c}390(43.3 \%) \\
414(45.9 \%) \\
97(10.8 \%) \\
(0.41)\end{array}$ & $\begin{array}{r}155(40.3 \%) \\
185(48.0 \%) \\
45(11.7 \%)\end{array}$ & $\begin{array}{c}365(41.2 \%) \\
396(44.6 \%) \\
126(14.2 \%) \\
(0.27)\end{array}$ & $\begin{array}{r}142(46.3 \%) \\
126(41.0 \%) \\
39(12.7 \%)\end{array}$ \\
\hline $\begin{array}{c}P R D M 2 \\
G \rightarrow A^{\ddagger}\end{array}$ & rs2235515 & $\begin{array}{c}G G \\
G A \\
A A \\
(0.23)\end{array}$ & $\begin{array}{c}1065(59.0 \%) \\
640(35.5 \%) \\
99(5.5 \%) \\
(0.82)\end{array}$ & $\begin{array}{c}377(55.7 \%) \\
262(38.7 \%) \\
38(5.6 \%)\end{array}$ & $\begin{array}{c}527(58.5 \%) \\
320(35.5 \%) \\
54(6.0 \%) \\
(0.56)\end{array}$ & $\begin{array}{c}210(56.0 \%) \\
141(37.6 \%) \\
24(6.4 \%)\end{array}$ & $\begin{array}{c}538(59.6 \%) \\
320(35.4 \%) \\
45(5.0 \%) \\
(0.77)\end{array}$ & $\begin{array}{c}167(55.3 \%) \\
121(40.1 \%) \\
14(4.6 \%)\end{array}$ \\
\hline
\end{tabular}

Abbreviations: SNP, single-nucleotide polymorphism; MAF, minor allele frequency among subcohort members (men and women combined); HWE, HardyWeinberg equilibrium; CRC, colorectal cancer.

${ }^{*} P$ value for test for Hardy-Weinberg equilibrium based on the distribution of genotypes among subcohort members.

${ }^{+} \chi^{2}$ Test; $P<0.05$ for the difference in genotype frequencies between colorectal cancer cases and subcohort members who did not develop colorectal cancer. ${ }^{\ddagger}$ Single-nucleotide polymorphism occurring in an intron of the gene.

the Methylation Specific PCR reaction $(38,39)$. All PCRs were done with controls for unmethylated alleles (DNA from normal lymphocytes), methylated alleles (normal lymphocyte DNA treated in vitro with SssI methyltransferase, New England Biolabs), and a control without DNA. Ten microliters of each Methylation Specific PCR reaction was directly loaded onto nondenaturing $6 \%$ polyacrylamide gels, stained with ethidium bromide, and visualized under UV illumination. The Methylation Specific PCR analyses were successful in $81 \%, 79 \%, 79 \%, 90 \%, 83 \%$, and $93 \%$ of the 734 patients for CACNA1G, IGF2, NEUROG1, RUNX3, SOCS1, and MLH1, respectively.

Microsatellite Instability. Microsatellite instability was determined by a pentaplex PCR, using the microsatellite instability markers BAT-26, BAT-25, NR-21, NR-22, and $N R-24$, as described in detail by Suraweera et al. (40). Microsatellite instability analyses were successful in 662 (90\%) of the 734 available samples.

Statistical Analyses. Data analyses were conducted overall and for men and women separately. The prevalence of the genotypes and minor allele frequencies of the single-nucleotide polymorphisms were calculated for subcohort members and colorectal cancer cases. $\chi^{2}$ Tests were used to test differences in prevalence between colorectal cancer cases and subcohort members who did not develop colorectal cancer. The Hardy-Weinberg equilibrium was tested using a $P$ value threshold of $<0.05$ among subcohort members to evaluate whether, for each singlenucleotide polymorphism, the individual alleles combined into the observed genotypes in a random manner. Although this subcohort was a random sample of the total cohort and thus selection would not be expected, the DNA samples were collected from individuals still alive several years after baseline. If survival of these subjects was due to presence of specific genotypes, this may bias the estimated associations. However, because genetic status is unknown of the remaining subcohort members, it is impossible to compare survival between genotypes, and we therefore alternatively compared mean age of patients between genotypes of each gene.

The overlap between the three methylation endpoints, that is, CIMP, MLH1 hypermethylation, and microsatellite instability, was compared and tested by $\chi^{2}$ tests. Cox proportional hazards regression models were used to estimate age-adjusted incidence rate ratios and 95\% 
confidence intervals (95\% CI), taking homozygotes of common alleles as reference category in all analyses. Analyses were done overall and stratified by CIMP status, MLH1 hypermethylation, and microsatellite instabil- ity, allowing us to compare the effects between the recently defined CIMP phenotype (36), the "classic" methylation marker MLH1, and microsatellite instability, and to account for a potential incomplete overlap that

Table 2. Associations of single-nucleotide polymorphisms in folate-metabolizing enzymes and epigenetic regulators with colorectal cancer risk

\begin{tabular}{|c|c|c|c|c|c|c|c|c|c|c|c|c|}
\hline \multirow[t]{2}{*}{ Gene and SNP } & \multirow[t]{2}{*}{ dbSNP number } & \multirow[t]{2}{*}{ Genotype } & \multicolumn{3}{|c|}{ Men and women } & \multicolumn{3}{|c|}{ Men } & \multicolumn{3}{|c|}{ Women } & \multirow{2}{*}{$\begin{array}{c}\begin{array}{c}\text { Interaction } \\
\text { with sex }\end{array} \\
P\end{array}$} \\
\hline & & & $n^{*}$ & RR $(95 \% \mathrm{CI})^{\dagger}$ & $P$ & $n$ & RR $(95 \% \mathrm{CI})^{\ddagger}$ & $P$ & $n$ & RR $(95 \% \text { CI })^{\ddagger}$ & $P$ & \\
\hline \multirow[t]{4}{*}{ MTHFR C677T } & \multirow[t]{4}{*}{ rs1801133 } & CC & 318 & 1.00 & 179 & 1.00 & 139 & 1.00 & & & & \\
\hline & & $C T$ & 320 & $\begin{array}{c}1.23 \\
(1.02-1.50)\end{array}$ & 0.04 & 184 & $\begin{array}{c}1.10 \\
(0.84-1.43)\end{array}$ & 0.49 & 136 & $\begin{array}{c}1.43 \\
(1.07-1.91)\end{array}$ & 0.02 & \\
\hline & & $T T$ & 51 & $\begin{array}{c}0.80 \\
(0.56-1.15)\end{array}$ & 0.23 & 19 & $\begin{array}{c}0.49 \\
(0.28-0.85)\end{array}$ & 0.01 & 32 & $\begin{array}{c}1.26 \\
(0.78-2.05)\end{array}$ & 0.34 & 0.03 \\
\hline & & $\begin{array}{c}C T+T T \\
\text { vs } C C\end{array}$ & 371 & $\begin{array}{c}1.15 \\
(0.95-1.39)\end{array}$ & 0.15 & 203 & $\begin{array}{c}0.98 \\
(0.76-1.27)\end{array}$ & 0.89 & 168 & $\begin{array}{c}1.39 \\
(1.06-1.83)\end{array}$ & 0.02 & \\
\hline \multirow[t]{4}{*}{ MTHFR A1298C } & \multirow[t]{4}{*}{ rs1801131 } & $A A$ & 299 & 1.00 & 167 & 1.00 & 132 & 1.00 & & & & \\
\hline & & $A C$ & 275 & $\begin{array}{c}0.89 \\
(0.72-1.09)\end{array}$ & 0.26 & 166 & $\begin{array}{c}0.81 \\
(0.62-1.07)\end{array}$ & 0.14 & 109 & $\begin{array}{c}1.00 \\
(0.73-1.35)\end{array}$ & 0.98 & \\
\hline & & $C C$ & 110 & $\begin{array}{c}1.05 \\
(0.79-1.38)\end{array}$ & 0.74 & 48 & $\begin{array}{c}0.89 \\
(0.59-1.35)\end{array}$ & 0.58 & 62 & $\begin{array}{c}1.23 \\
(0.85-1.78)\end{array}$ & 0.28 & 0.42 \\
\hline & & $\begin{array}{c}A C+C C \\
\text { vs } A A\end{array}$ & 385 & $\begin{array}{c}0.93 \\
(0.77-1.12)\end{array}$ & 0.45 & 214 & $\begin{array}{c}0.83 \\
(0.64-1.07)\end{array}$ & 0.15 & 171 & $\begin{array}{c}1.07 \\
(0.81-1.41)\end{array}$ & 0.64 & \\
\hline \multirow[t]{3}{*}{ MTR A2756G } & \multirow[t]{3}{*}{ rs1805087 } & $\begin{array}{l}A A \\
A G\end{array}$ & $\begin{array}{l}449 \\
204\end{array}$ & $\begin{array}{l}1.00 \\
1.03\end{array}$ & $\begin{array}{l}257 \\
0.77\end{array}$ & $\begin{array}{l}1.00 \\
108\end{array}$ & $\begin{array}{l}192 \\
0.95\end{array}$ & $\begin{array}{l}1.00 \\
0.73\end{array}$ & 96 & 1.14 & 0.38 & \\
\hline & & GG & 43 & 1.58 & 0.04 & 22 & $\begin{array}{c}(0 . / 2-1.26) \\
1.45\end{array}$ & 0.23 & 21 & $\left(\begin{array}{l}(0.05-1.34) \\
1.76\end{array}\right.$ & 0.06 & 0.64 \\
\hline & & $\begin{array}{c}A G+G G \\
\text { vs } A A\end{array}$ & 247 & $\begin{array}{c}(1.03-2.43) \\
1.10 \\
(0.90-1.33)\end{array}$ & 0.35 & 130 & $\begin{array}{c}(0.79-2.66) \\
1.01 \\
(0.77-1.32)\end{array}$ & 0.94 & 117 & $\begin{array}{c}(0.97-3.21) \\
1.22 \\
(0.92-1.62)\end{array}$ & 0.17 & \\
\hline \multirow[t]{3}{*}{ MTRR A66G } & \multirow[t]{3}{*}{ rs1801394 } & $\begin{array}{l}A A \\
A G\end{array}$ & $\begin{array}{l}136 \\
338\end{array}$ & $\begin{array}{c}1.00 \\
1.08 \\
(0.85-1.39)\end{array}$ & $\begin{array}{l}65 \\
0.53\end{array}$ & $\begin{array}{l}1.00 \\
196\end{array}$ & $\begin{array}{c}71 \\
1.36 \\
(0.96-1.92)\end{array}$ & $\begin{array}{l}1.00 \\
0.09\end{array}$ & 142 & $\begin{array}{c}0.83 \\
(0.59-1.18)\end{array}$ & 0.31 & \\
\hline & & GG & 225 & $\begin{array}{c}1.03 \\
(0.79-1.34)\end{array}$ & 0.83 & 129 & $\begin{array}{c}1.23 \\
(0.85-1.78)\end{array}$ & 0.27 & 96 & $\begin{array}{c}0.85 \\
(0.58-1.24)\end{array}$ & 0.39 & 0.15 \\
\hline & & $\begin{array}{c}A G+G G \\
\quad \text { vs } A A\end{array}$ & 563 & $\begin{array}{c}1.06 \\
(0.84-1.34)\end{array}$ & 0.62 & 325 & $\begin{array}{c}1.30 \\
(0.94-1.81)\end{array}$ & 0.11 & 238 & $\begin{array}{c}0.84 \\
(0.60-1.16)\end{array}$ & 0.29 & \\
\hline \multirow[t]{4}{*}{ DNMT3b $C \rightarrow T$} & \multirow[t]{4}{*}{ rs2424913 } & $C C$ & 240 & 1.00 & 132 & 1.00 & 108 & 1.00 & & & & \\
\hline & & $C T$ & 348 & $\begin{array}{c}0.95 \\
(0.78-1.17)\end{array}$ & 0.66 & 184 & $\begin{array}{c}0.91 \\
(0.68-1.20)\end{array}$ & 0.50 & 164 & $\begin{array}{c}1.01 \\
(0.75-1.36)\end{array}$ & 0.94 & \\
\hline & & $T T$ & 115 & $\begin{array}{l}0.90 \\
(0.68-1.19)\end{array}$ & 0.46 & 73 & $\begin{array}{c}1.00 \\
(0.69-1.45)\end{array}$ & 0.99 & 42 & $\begin{array}{c}0.76 \\
(0.50-1.16)\end{array}$ & 0.20 & 0.37 \\
\hline & & $\begin{array}{c}C T+T T \\
\text { vs } C C\end{array}$ & 463 & $\begin{array}{c}0.94 \\
(0.77-1.14)\end{array}$ & 0.54 & 257 & $\begin{array}{c}0.93 \\
(0.71-1.22)\end{array}$ & 0.60 & 206 & $\begin{array}{c}0.95 \\
(0.71-1.26)\end{array}$ & 0.72 & \\
\hline \multirow[t]{3}{*}{ DNMT3b $C \rightarrow T$} & \multirow[t]{3}{*}{ rs406193 } & $\begin{array}{l}C C \\
C T\end{array}$ & $\begin{array}{l}528 \\
152\end{array}$ & $\begin{array}{c}1.00 \\
0.91 \\
(0.73-1.14)\end{array}$ & $\begin{array}{l}291 \\
0.43\end{array}$ & $\begin{array}{c}1.00 \\
88\end{array}$ & $\begin{array}{c}237 \\
1.13 \\
(0.83-1.54)\end{array}$ & $\begin{array}{l}1.00 \\
0.43\end{array}$ & 64 & $\begin{array}{c}0.71 \\
(0.51-0.99)\end{array}$ & 0.05 & \\
\hline & & $\mathrm{TT}$ & 18 & $\begin{array}{c}1.05 \\
(0.59-1.87)\end{array}$ & 0.87 & 11 & $\begin{array}{c}1.32 \\
(0.59-2.95)\end{array}$ & 0.50 & 7 & - & - & 0.10 \\
\hline & & $\begin{array}{c}C T+T T \\
\text { vs } C C\end{array}$ & 170 & $\begin{array}{c}0.93 \\
(0.75-1.15)\end{array}$ & 0.49 & 99 & $\begin{array}{c}1.15 \\
(0.86-1.54)\end{array}$ & 0.36 & 71 & $\begin{array}{c}0.72 \\
(0.52-0.99)\end{array}$ & 0.04 & \\
\hline \multirow[t]{2}{*}{ EHMT1 G $\rightarrow A$} & \multirow[t]{2}{*}{ rs4634736 } & $\begin{array}{l}G G \\
G A\end{array}$ & $\begin{array}{l}568 \\
121\end{array}$ & $\begin{array}{c}1.00 \\
0.93 \\
(0.73-1.18)\end{array}$ & $\begin{array}{l}320 \\
0.55\end{array}$ & $\begin{array}{c}1.00 \\
64\end{array}$ & $\begin{array}{c}248 \\
0.92 \\
(0.66-1.28)\end{array}$ & $\begin{array}{l}1.00 \\
0.62\end{array}$ & 57 & $\begin{array}{c}0.95 \\
(0.67-1.34)\end{array}$ & 0.76 & \\
\hline & & $\begin{array}{l}A A \\
G A+A A \\
\quad \text { vs } G G\end{array}$ & $\begin{array}{r}6 \\
127\end{array}$ & $\begin{array}{c}\overline{0.93} \\
(0.73-1.18)\end{array}$ & $\overline{0.55}$ & $\begin{array}{r}1 \\
65\end{array}$ & $\begin{array}{c}\overline{0.89} \\
(0.64-1.24)\end{array}$ & $\overline{0.49}$ & $\begin{array}{r}5 \\
62\end{array}$ & $\begin{array}{c}\overline{0.98} \\
(0.70-1.37)\end{array}$ & $\overline{0.90}$ & 0.41 \\
\hline \multirow[t]{3}{*}{ EHMT2 $G \rightarrow A$} & \multirow[t]{3}{*}{ rs535586 } & $\begin{array}{l}G G \\
G A\end{array}$ & $\begin{array}{l}297 \\
311\end{array}$ & $\begin{array}{c}1.00 \\
0.92 \\
(0.76-1.13)\end{array}$ & $\begin{array}{l}155 \\
0.44\end{array}$ & $\begin{array}{l}1.00 \\
185\end{array}$ & $\begin{array}{c}142 \\
1.10 \\
(0.84-1.44)\end{array}$ & $\begin{array}{l}1.00 \\
0.48\end{array}$ & 126 & $\begin{array}{c}0.74 \\
(0.55-0.99)\end{array}$ & 0.05 & \\
\hline & & $A A$ & 84 & $\begin{array}{c}1.01 \\
(0.74-1.37)\end{array}$ & 0.95 & 45 & $\begin{array}{c}1.17 \\
(0.76-1.80)\end{array}$ & 0.47 & 39 & $\begin{array}{c}0.84 \\
(0.55-1.27)\end{array}$ & 0.44 & 0.13 \\
\hline & & $\begin{array}{c}G A+A A \\
\text { vs } G G\end{array}$ & 395 & $\begin{array}{c}0.94 \\
(0.78-1.14)\end{array}$ & 0.53 & 230 & $\begin{array}{c}1.12 \\
(0.86-1.44)\end{array}$ & 0.41 & 165 & $\begin{array}{c}0.76 \\
(0.58-1.01)\end{array}$ & 0.05 & \\
\hline \multirow[t]{3}{*}{ PRDM2 G $\rightarrow A$} & \multirow[t]{3}{*}{ rs2235515 } & $\begin{array}{l}G G \\
G A\end{array}$ & $\begin{array}{l}377 \\
262\end{array}$ & $\begin{array}{c}1.00 \\
1.14 \\
(0.93-1.38)\end{array}$ & $\begin{array}{l}210 \\
0.20\end{array}$ & $\begin{array}{l}1.00 \\
141\end{array}$ & $\begin{array}{c}167 \\
1.09 \\
(0.83-1.43)\end{array}$ & $\begin{array}{l}1.00 \\
0.54\end{array}$ & 121 & $\begin{array}{c}1.20 \\
(0.90-1.60)\end{array}$ & 0.21 & \\
\hline & & $A A$ & 38 & $\begin{array}{c}1.09 \\
(0.72-1.66)\end{array}$ & 0.67 & 24 & $\begin{array}{c}1.02 \\
(0.60-1.75)\end{array}$ & 0.93 & 14 & $\begin{array}{c}1.20 \\
(0.63-2.29)\end{array}$ & 0.58 & 0.85 \\
\hline & & $\begin{array}{c}G A+A A \\
\text { vs } G G\end{array}$ & 300 & $\begin{array}{c}1.13 \\
(0.94-1.37)\end{array}$ & 0.20 & 165 & $\begin{array}{c}1.08 \\
(0.83-1.40)\end{array}$ & 0.57 & 135 & $\begin{array}{c}1.20 \\
(0.91-1.58)\end{array}$ & 0.20 & \\
\hline
\end{tabular}

Abbreviation: $R R$, incidence rate ratio.

*Number of cases; subgroups of $\leq 10$ cases were considered too small for precise estimates and were therefore omitted from the table.

${ }^{+}$Adjusted for age and sex.

${ }^{\ddagger}$ Adjusted for age. 
Table 3. Frequency of colorectal cancers harboring CIMP and overlap with MLH1 promoter hypermethylation and microsatellite instability

\begin{tabular}{lccc}
\hline & $\begin{array}{c}\text { Frequency of molecular } \\
\text { phenotype, } n(\%)\end{array}$ & CIMP & CIMP- \\
\cline { 3 - 4 } & CIMP & CIMP+ & - \\
CIMP+ & $167(27.7)$ & - & - \\
CIMP- & $436(72.3)$ & $68(41.5)$ & $59(14.0)$ \\
MLH1 promoter methylation & $152(22.1)$ & $96(58.5)$ & $363(86.0)$ \\
$\quad$ Methylated & $534(77.9)$ & $52(33.3)$ & $17(4.2)$ \\
MSI & $84(12.7)$ & $104(67.7)$ & $390(95.8)$
\end{tabular}

Abbreviations: CIMP+; three or more of five CIMP markers methylated; CIMP-, zero to two of five CIMP markers methylated; MSI, microsatellite instability; MSS, Microsatellite Stable tumors.

${ }^{*} \chi^{2}$ Test.

may exist between related molecular phenotypes (41). Because two single-nucleotide polymorphisms were determined for MTHFR and DNMT3b, we estimated incidence rate ratios for combinations of genotypes within these genes. In addition, incidence rate ratios for combinations of genotypes per functional group (that is, based on the number of rare alleles in any of the folate-metabolizing enzymes MTHFR, MTR, and MTRR, or in any of the histone methyltransferases EHMT1, EHMT2, and $P R D M 2)$ or the combination of all the studied genes were estimated. SEs of the incidence rate ratios were estimated using the robust Huber-White sandwich estimator to account for additional variance introduced by sampling from the cohort (42). The proportional hazards assumption was tested using the scaled Schoenfeld residuals (43). Multivariate-adjusted analyses were additionally conducted, including the covariates dietary folate, vitamin B2, vitamin B6, methionine, alcohol, energy intake, family history of colorectal cancer, smoking behavior, and body mass index. Interactions with sex were tested for each of the single-nucleotide polymorphisms. Statistical analyses were done with the Stata statistical software package (version 10).

\section{Results}

The overall and gender-specific prevalences of genotypes were calculated and compared between subcohort members and colorectal cancer cases. We observed that the rare MTHFR 677TT variant occurred more often in subcohort members than in cases and that this difference was present among men (prevalences in subcohort and cases were $9.7 \%$ and $5.0 \%$, respectively). Prevalences of other genotypes did not significantly differ between subcohort members and cases (Table 1). The distribution of the MTHFR A1298C, MTRR A66G, and DNMT3b C $\rightarrow$ T (rs406193) genotypes deviated from the Hardy-Weinberg equilibrium $(P<0.05)$. However, mean age was similar between genotypes of any of the single-nucleotide polymorphisms, suggesting that there was no survivorship effect due to genetic status.

Overall, subjects with the heterozygous MTHFR $677 C T$ genotype were at modestly increased colorectal cancer risk compared with participants with the common $C C$ genotype (incidence rate ratio, $1.23 ; P=0.04$; Table 2). Similarly, positive associations existed among women with this genotype (incidence rate ratio, 1.43; $P=0.02$ ) and for the occurrence of the rare $T$ allele (that is, the combination of the $C T$ and $T T$ genotypes compared with $C C$; incidence rate ratio, $1.39 ; P=0.02$ ). Conversely, we observed that men homozygous for the MTHFR 677TT genotype were at reduced colorectal cancer risk (incidence rate ratio, $0.49 ; P=0.01$ ). There was a significant interaction between sex and MTHFR C677T genotypes $(P=0.03)$. Whereas MTHFR A1298C was not associated with colorectal cancer, we observed that the MTR 2756GG genotype was associated with increased risk for colorectal cancer (incidence rate ratio, 1.58; $P=0.04$ ), particularly among women (incidence rate ratio, $1.76 ; P=0.06$ ). Among men, the MTR 2756GG genotype was associated with a nonsignificant increase of colorectal cancer risk (incidence rate ratio, $1.45 ; P=0.23)$. MTRR A66G genotypes were not associated with colorectal cancer.

Among women, compared with common homozygotes, the rare alleles of DNMT3b $C \rightarrow T$ (rs406193) and EHMT2 $G \rightarrow A$ (rs535586) were associated with decreased colorectal cancer risk [incidence rate ratio, 0.72 $(P=0.04)$ and $0.76(P=0.05)$, respectively], but such associations were not observed among men. No associations were observed between individual genotypes of the remaining genes and overall colorectal cancer risk. Combining MTHFR and DNMT3b genotypes, genotypes

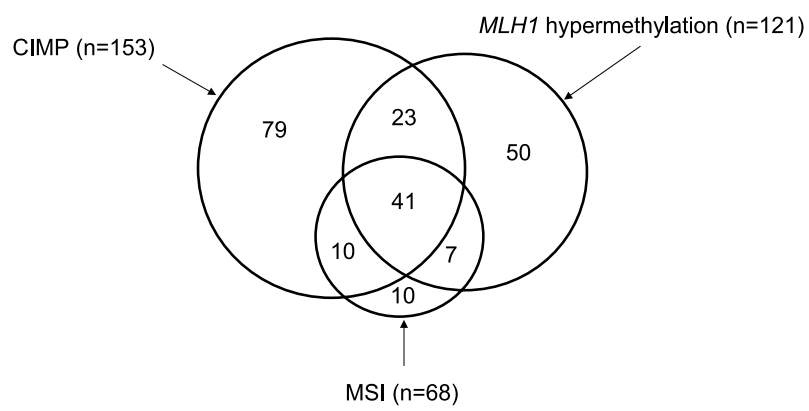

Figure 2. Overlap between CIMP, MLH1 hypermethylation, and microsatellite instability in colorectal cancers, showing at least one of these three aberrations. In total, there were $n=271$ colorectal cancers harboring at least one aberration. Numbers are based on colorectal cancers with complete analyses of all three molecular characteristics $(n=547$ colorectal cancers). The sizes of the different areas in this figure may not exactly reflect the numbers of the applicable subsets. 
per functional group, or genotypes of all studied genes did not reveal any clear associations with colorectal cancer. Multivariate-adjusted analyses resulted in similar findings compared with age-adjusted analyses (data not shown).

In total, $167(27.7 \%)$ of the 603 colorectal cancers with available CIMP analyses showed the CIMP phenotype (Table 3; Fig. 2), with no significant difference between men and women (data not shown). As reported previously for MLH1 hypermethylation, microsatellite instability, and BRAF mutations (41), the overlap between CIMP, MLH1 hypermethylation, and microsatellite instability was also incomplete. Although statistically significant $\left(P_{\chi^{2}}<0.001\right), 41.5 \%$ of the colorectal cancers with CIMP showed MLH1 methylation, whereas only $33.3 \%$ harbored microsatellite instability.

MTHFR C677T polymorphisms were not associated with any of the methylation endpoints among men (Table 4). However, we observed that women carrying the rare T allele of MTHFR C677T were at significantly increased risk for developing a tumor without CIMP (CIMP-; incidence rate ratio, 1.40; $P=0.04)$, without $M L H 1$ hypermethylation (incidence rate ratio, $1.49 ; P=0.01$ ), or without microsatellite instability (incidence rate ratio, 1.36; $P=0.04$ ) but also those with CIMP (CIMP+; incidence rate ratio, $1.65 ; P=0.04$; Table 5 ). Among men, the rare $C$ allele of the MTHFR $A 1298 \mathrm{C}$ polymorphism was inversely associated with colorectal cancers without CIMP (incidence rate ratio, $0.72 ; P=0.03$ ). Inverse associations were also observed between MTR A2756G and
CIMP+ among men (incidence rate ratio, $0.58 ; P=0.04$ ) and between MTRR A66G and MLH1 hypermethylation among women (incidence rate ratio, $0.55 ; P=0.02$ ).

Among women, the rare $T$ allele of DNMT3b $C \rightarrow T$ (rs406193) was associated with decreased risks for colorectal cancers without CIMP (incidence rate ratio, 0.67; $P=0.04)$, colorectal cancers without microsatellite instability (incidence rate ratio, $0.70 ; P=0.04$ ), and a nonsignificant decreased risk for colorectal cancers without MLH1 hypermethylation (incidence rate ratio, 0.73; $P=0.09$ ). However, although not statistically significant, incidence rate ratios were also decreased for colorectal cancers harboring CIMP or MLH1 hypermethylation. Similarly, the rare A allele of $E H M T 2 \mathrm{G} \rightarrow A$ (rs535586) was inversely associated colorectal cancers without MLH1 methylation (incidence rate ratio, 0.73), colorectal cancers without microsatellite instability (incidence rate ratio, 0.73 ), or nonsignificantly with colorectal cancers without CIMP (incidence rate ratio, 0.75) in women. Conversely, we observed a positive association (incidence rate ratio, 1.99) of $E H M T 2 \mathrm{G} \rightarrow A$ with $M L H 1$ hypermethylation among men.

\section{Discussion}

We studied associations between single-nucleotide polymorphisms in folate metabolizing enzymes, a DNA methyltransferase, and histone methyltransferases with colorectal cancer risk, accounting for related "methylation phenotypes" in a large prospective cohort study in the

Table 4. Associations of single-nucleotide polymorphisms in folate metabolizing enzymes and epigenetic regulators with colorectal cancer risk according to methylation status of the tumor among men

\begin{tabular}{|c|c|c|c|c|c|c|c|c|c|c|c|c|c|}
\hline \multirow[t]{3}{*}{ Gene and SNP } & \multirow[t]{3}{*}{ Genotype } & \multicolumn{4}{|c|}{ CIMP } & \multicolumn{4}{|c|}{ MLH1 promoter hypermethylation } & \multicolumn{4}{|c|}{ MSI } \\
\hline & & \multicolumn{2}{|r|}{ CIMP+ } & \multicolumn{2}{|r|}{ CIMP- } & \multicolumn{2}{|r|}{ Methylated } & \multicolumn{2}{|c|}{ Unmethylated } & \multicolumn{2}{|r|}{ MSI } & \multicolumn{2}{|r|}{ No MSI } \\
\hline & & $n^{*}$ & $\operatorname{RR}(95 \% \mathrm{CI})^{+}$ & $n \mathrm{I}$ & $\mathrm{RR}(95 \% \mathrm{CI})^{+}$ & $n \mathrm{I}$ & $\mathrm{RR}(95 \% \mathrm{CI})^{+}$ & $n$ & $\mathrm{RR}(95 \% \mathrm{CI})^{\dagger}$ & $n \mathrm{I}$ & $\operatorname{RR}(95 \% \mathrm{CI})^{+}$ & $n$ & $\operatorname{RR}(95 \% \mathrm{CI})^{+}$ \\
\hline $\begin{array}{l}\text { ITHFR C677T } \\
\text { s1801133) }\end{array}$ & $\begin{array}{l}C C \\
C T+T T\end{array}$ & $\begin{array}{l}43 \\
40\end{array}$ & $\begin{array}{c}\text { Ref. } \\
0.81 \\
(0.51-1.28)\end{array}$ & $\begin{array}{l}103 \\
135\end{array}$ & $\begin{array}{c}\text { Ref. } \\
1.14 \\
(0.84-1.54)\end{array}$ & $\begin{array}{l}32 \\
41\end{array}$ & $\begin{array}{c}\text { Ref. } \\
1.11 \\
(0.68-1.81)\end{array}$ & $\begin{array}{l}143 \\
153\end{array}$ & $\begin{array}{c}\text { Ref. } \\
0.93 \\
(0.70-1.22)\end{array}$ & $\begin{array}{l}21 \\
20\end{array}$ & $\begin{array}{c}\text { Ref. } \\
0.83 \\
(0.44-1.58)\end{array}$ & $\begin{array}{l}145 \\
166\end{array}$ & $\begin{array}{c}\text { Ref. } \\
0.99 \\
(0.75-1.30)\end{array}$ \\
\hline $\begin{array}{l}\text { MTHFR A1298C } \\
\text { (rs1801131) }\end{array}$ & $\begin{array}{l}A A \\
A C+C C\end{array}$ & $\begin{array}{l}30 \\
54\end{array}$ & $\begin{array}{l}\text { Ref. } \\
1.16 \\
(0.72-1.87)\end{array}$ & $\begin{array}{l}112 \\
125\end{array}$ & $\begin{array}{l}\text { Ref. } \\
0.72 \\
(0.53-0.97)\end{array}$ & $\begin{array}{l}32 \\
40\end{array}$ & $\begin{array}{l}\text { Ref. } \\
0.81 \\
(0.49-1.32)\end{array}$ & $\begin{array}{l}132 \\
165\end{array}$ & $\begin{array}{l}\text { Ref. } \\
0.81 \\
(0.61-1.07)\end{array}$ & $\begin{array}{l}16 \\
24\end{array}$ & $\begin{array}{l}\text { Ref. } \\
0.97 \\
(0.50-1.88)\end{array}$ & $\begin{array}{l}139 \\
173\end{array}$ & $\begin{array}{l}\text { Ref. } \\
0.80 \\
(0.61-1.06)\end{array}$ \\
\hline $\begin{array}{l}\text { ITR A2756G } \\
\text { s1805087) }\end{array}$ & $\begin{array}{l}A A \\
A G+G G\end{array}$ & $\begin{array}{l}66 \\
19\end{array}$ & $\begin{array}{l}\text { Ref. } \\
0.58 \\
(0.34-0.99)\end{array}$ & $\begin{array}{r}153 \\
87\end{array}$ & $\begin{array}{l}\text { Ref. } \\
1.14 \\
(0.83-1.55)\end{array}$ & $\begin{array}{l}49 \\
24\end{array}$ & $\begin{array}{l}\text { Ref. } \\
0.98 \\
(0.58-1.65)\end{array}$ & $\begin{array}{l}199 \\
100\end{array}$ & $\begin{array}{l}\text { Ref. } \\
1.01 \\
(0.75-1.35)\end{array}$ & $\begin{array}{l}25 \\
16\end{array}$ & $\begin{array}{l}\text { Ref. } \\
1.31 \\
(0.68-2.51)\end{array}$ & $\begin{array}{l}209 \\
107\end{array}$ & $\begin{array}{l}\text { Ref. } \\
1.02 \\
(0.77-1.36)\end{array}$ \\
\hline $\begin{array}{l}\text { ITRR A66G } \\
\text { s1801394) }\end{array}$ & $\begin{array}{l}A A \\
A G+G G\end{array}$ & $\begin{array}{l}10 \\
75\end{array}$ & Ref. & $\begin{array}{r}45 \\
197\end{array}$ & $\begin{array}{l}\text { Ref. } \\
1.14 \\
(0.79-1.66)\end{array}$ & $\begin{array}{l}12 \\
61\end{array}$ & $\begin{array}{l}\text { Ref. } \\
1.32 \\
(0.69-2.52)\end{array}$ & $\begin{array}{r}49 \\
252\end{array}$ & $\begin{array}{l}\text { Ref. } \\
1.34 \\
(0.93-1.92)\end{array}$ & $\begin{array}{r}5 \\
36\end{array}$ & Ref. & $\begin{array}{r}56 \\
262\end{array}$ & $\begin{array}{l}\text { Ref. } \\
1.22 \\
(0.87-1.72)\end{array}$ \\
\hline$C \rightarrow T$ & $\begin{array}{l}C C \\
C T+T T\end{array}$ & $\begin{array}{l}29 \\
56\end{array}$ & $\begin{array}{l}\text { Ref. } \\
0.92 \\
(0.60-1.49)\end{array}$ & $\begin{array}{r}81 \\
161\end{array}$ & $\begin{array}{l}\text { Ref. } \\
0.95 \\
(0.69-1.30)\end{array}$ & $\begin{array}{l}31 \\
42\end{array}$ & $\begin{array}{l}\text { Ref. } \\
0.65 \\
(0.39-1.06)\end{array}$ & $\begin{array}{r}95 \\
205\end{array}$ & $\begin{array}{l}\text { Ref. } \\
1.03 \\
(0.77-1.38)\end{array}$ & $\begin{array}{l}17 \\
24\end{array}$ & $\begin{array}{c}\text { Ref. } \\
0.66 \\
(0.35-1.28)\end{array}$ & $\begin{array}{l}101 \\
216\end{array}$ & $\begin{array}{l}\text { Ref. } \\
1.03 \\
(0.77-1.36)\end{array}$ \\
\hline $\begin{array}{l}\text { DNMT3b C } \rightarrow T \\
\text { (rs406193) }\end{array}$ & $\begin{array}{l}C C \\
C T+T T\end{array}$ & $\begin{array}{l}59 \\
26\end{array}$ & $\begin{array}{l}\text { Ref. } \\
1.49 \\
(0.90-2.45)\end{array}$ & $\begin{array}{r}182 \\
60\end{array}$ & $\begin{array}{l}\text { Ref. } \\
1.11 \\
(0.79-1.57)\end{array}$ & $\begin{array}{l}54 \\
19\end{array}$ & $\begin{array}{l}\text { Ref. } \\
1.19 \\
(0.68-2.08)\end{array}$ & $\begin{array}{r}223 \\
78\end{array}$ & $\begin{array}{l}\text { Ref. } \\
1.18 \\
(0.86-1.62)\end{array}$ & $\begin{array}{l}30 \\
11\end{array}$ & $\begin{array}{l}\text { Ref. } \\
1.25 \\
(0.61-2.56)\end{array}$ & $\begin{array}{r}239 \\
79\end{array}$ & $\begin{array}{l}\text { Ref. } \\
1.12 \\
(0.82-1.53)\end{array}$ \\
\hline $\begin{array}{l}\text { EHMT1 G } \rightarrow \text { (rs4634736) } \\
\rightarrow\end{array}$ & $\begin{array}{l}G G \\
G A+A A\end{array}$ & $\begin{array}{l}74 \\
11\end{array}$ & $\begin{array}{l}\text { Ref. } \\
0.65 \\
(0.34-1.26)\end{array}$ & $\begin{array}{r}197 \\
43\end{array}$ & $\begin{array}{l}\text { Ref. } \\
0.96 \\
(0.65-1.40)\end{array}$ & $\begin{array}{l}57 \\
16\end{array}$ & $\begin{array}{l}\text { Ref. } \\
1.23 \\
(0.69-2.22)\end{array}$ & $\begin{array}{r}253 \\
46\end{array}$ & $\begin{array}{l}\text { Ref. } \\
0.84 \\
(0.58-1.21)\end{array}$ & $\begin{array}{r}36 \\
4\end{array}$ & Ref. & $\begin{array}{r}259 \\
57\end{array}$ & $\begin{array}{l}\text { Ref. } \\
0.96 \\
(0.68-1.36)\end{array}$ \\
\hline $\begin{array}{l}\text { EHMT2 G } \rightarrow A \\
(\text { rs535586) }\end{array}$ & $\begin{array}{l}G G \\
G A+A A\end{array}$ & $\begin{array}{l}34 \\
50\end{array}$ & $\begin{array}{l}\text { Ref. } \\
1.11 \\
(0.69-1.76)\end{array}$ & $\begin{array}{r}98 \\
143\end{array}$ & $\begin{array}{l}\text { Ref. } \\
1.10 \\
(0.81-1.48)\end{array}$ & $\begin{array}{l}20 \\
53\end{array}$ & $\begin{array}{l}\text { Ref. } \\
1.99 \\
(1.16-3.41)\end{array}$ & $\begin{array}{l}130 \\
168\end{array}$ & $\begin{array}{l}\text { Ref. } \\
0.97 \\
(0.73-1.28)\end{array}$ & $\begin{array}{l}15 \\
26\end{array}$ & $\begin{array}{l}\text { Ref. } \\
1.30 \\
(0.67-2.51)\end{array}$ & $\begin{array}{l}124 \\
190\end{array}$ & $\begin{array}{l}\text { Ref. } \\
1.15 \\
(0.88-1.52)\end{array}$ \\
\hline $\begin{array}{l}P R D M 2 ~ G \rightarrow A \\
(\mathrm{rs} 2235515)\end{array}$ & $\begin{array}{l}G G \\
G A+A A\end{array}$ & $\begin{array}{l}46 \\
36\end{array}$ & $\begin{array}{l}\text { Ref. } \\
1.07 \\
(0.67-1.71)\end{array}$ & $\begin{array}{l}126 \\
109\end{array}$ & $\begin{array}{l}\text { Ref. } \\
1.19 \\
(0.88-1.60)\end{array}$ & $\begin{array}{l}41 \\
31\end{array}$ & $\begin{array}{l}\text { Ref. } \\
1.04 \\
(0.63-1.71)\end{array}$ & $\begin{array}{l}160 \\
130\end{array}$ & $\begin{array}{l}\text { Ref. } \\
1.11 \\
(0.84-1.47)\end{array}$ & $\begin{array}{l}47 \\
34\end{array}$ & $\begin{array}{l}\text { Ref. } \\
0.73 \\
(0.37-1.44)\end{array}$ & $\begin{array}{l}166 \\
140\end{array}$ & $\begin{array}{l}\text { Ref. } \\
1.16 \\
(0.88-1.52)\end{array}$ \\
\hline
\end{tabular}

Abbreviation: Ref., reference.

*Number of cases; subgroups of $\leq 10$ cases were considered too small for precise estimates and were therefore omitted from the table.

${ }^{+}$Age-adjusted incidence rate ratios $(95 \% \mathrm{CI})$.

$\ddagger$ dbSNP number. 
Table 5. Associations of single-nucleotide polymorphisms in folate-metabolizing enzymes and epigenetic regulators with colorectal cancer risk according to methylation status of the tumor among women

\begin{tabular}{|c|c|c|c|c|c|c|c|c|c|c|c|c|c|}
\hline \multirow[t]{3}{*}{ Gene and SNP } & \multirow[t]{3}{*}{ Genotype } & \multicolumn{4}{|c|}{ CIMP } & \multicolumn{4}{|c|}{ MLH1 promoter hypermethylation } & \multicolumn{4}{|c|}{ MSI } \\
\hline & & \multicolumn{2}{|r|}{ CIMP+ } & \multicolumn{2}{|r|}{ CIMP- } & \multicolumn{2}{|r|}{ Methylated } & \multicolumn{2}{|c|}{ Unmethylated } & \multicolumn{2}{|r|}{ MSI } & \multicolumn{2}{|r|}{ No MSI } \\
\hline & & $n^{*}$ & $\operatorname{RR}(95 \% \mathrm{CI})^{\dagger}$ & $n$ & RR $(95 \%$ CI $)$ & $n$ & $\mathrm{RR}(95 \% \mathrm{CI})$ & $n$ & $\operatorname{RR}(95 \% \mathrm{CI})$ & $n$ & $\mathrm{RR}(95 \% \mathrm{CI})$ & $n$ & $\mathrm{RR}(95 \% \mathrm{CI})$ \\
\hline $\begin{array}{l}\text { MTHFR C677T } \\
\text { (rs1801133) }^{\ddagger}\end{array}$ & $\begin{array}{l}C C \\
C T+T T\end{array}$ & $\begin{array}{l}33 \\
47\end{array}$ & $\begin{array}{c}\text { Ref. } \\
1.65 \\
(1.02-2.65)\end{array}$ & $\begin{array}{r}82 \\
100\end{array}$ & $\begin{array}{c}\text { Ref. } \\
1.40 \\
(1.01-1.94)\end{array}$ & $\begin{array}{l}41 \\
37\end{array}$ & $\begin{array}{c}\text { Ref. } \\
1.03 \\
(0.64-1.65)\end{array}$ & $\begin{array}{r}94 \\
121\end{array}$ & $\begin{array}{c}\text { Ref. } \\
1.49 \\
(1.09-2.03)\end{array}$ & $\begin{array}{l}18 \\
23\end{array}$ & $\begin{array}{c}\text { Ref. } \\
1.49 \\
(0.78-2.84)\end{array}$ & $\begin{array}{l}115 \\
136\end{array}$ & $\begin{array}{c}\text { Ref. } \\
1.36 \\
(1.02-1.82)\end{array}$ \\
\hline $\begin{array}{l}\text { MTHFR A1298C } \\
\text { (rs1801131) }\end{array}$ & $\begin{array}{l}A A \\
A C+C C\end{array}$ & $\begin{array}{l}34 \\
46\end{array}$ & $\begin{array}{l}\text { Ref. } \\
1.12 \\
(0.70-1.80)\end{array}$ & $\begin{array}{l}80 \\
99\end{array}$ & $\begin{array}{l}\text { Ref. } \\
1.01 \\
(0.73-1.41)\end{array}$ & $\begin{array}{l}32 \\
45\end{array}$ & $\begin{array}{l}\text { Ref. } \\
1.15 \\
(0.71-1.85)\end{array}$ & $\begin{array}{r}92 \\
120\end{array}$ & $\begin{array}{l}\text { Ref. } \\
1.08 \\
(0.79-1.48)\end{array}$ & $\begin{array}{l}22 \\
19\end{array}$ & $\begin{array}{l}\text { Ref. } \\
0.73 \\
(0.38-1.39)\end{array}$ & $\begin{array}{l}102 \\
146\end{array}$ & $\begin{array}{l}\text { Ref. } \\
1.18 \\
(0.87-1.59)\end{array}$ \\
\hline $\begin{array}{l}\text { MTR A2756G } \\
\text { (rs1805087) }\end{array}$ & $\begin{array}{l}A A \\
A G+G G\end{array}$ & $\begin{array}{l}54 \\
26\end{array}$ & $\begin{array}{l}\text { Ref. } \\
0.96 \\
(0.59-1.58)\end{array}$ & $\begin{array}{r}115 \\
70\end{array}$ & $\begin{array}{l}\text { Ref. } \\
1.22 \\
(0.87-1.71)\end{array}$ & $\begin{array}{l}47 \\
31\end{array}$ & $\begin{array}{l}\text { Ref. } \\
1.32 \\
(0.82-2.13)\end{array}$ & $\begin{array}{r}137 \\
60\end{array}$ & $\begin{array}{l}\text { Ref. } \\
1.17 \\
(0.85-2.61)\end{array}$ & $\begin{array}{l}25 \\
16\end{array}$ & $\begin{array}{l}\text { Ref. } \\
1.28 \\
(0.67-2.45)\end{array}$ & $\begin{array}{r}156 \\
98\end{array}$ & $\begin{array}{l}\text { Ref. } \\
1.26 \\
(0.93-1.70)\end{array}$ \\
\hline $\begin{array}{l}\text { MTRR A66G } \\
\text { (rs1801394) }\end{array}$ & $\begin{array}{l}A A \\
A G+G G\end{array}$ & $\begin{array}{l}16 \\
64\end{array}$ & $\begin{array}{l}\text { Ref. } \\
1.00 \\
(0.56-1.79)\end{array}$ & $\begin{array}{r}46 \\
139\end{array}$ & $\begin{array}{l}\text { Ref. } \\
0.75 \\
(0.51-1.11)\end{array}$ & $\begin{array}{l}24 \\
53\end{array}$ & $\begin{array}{l}\text { Ref. } \\
0.55 \\
(0.33-0.92)\end{array}$ & $\begin{array}{r}44 \\
252\end{array}$ & $\begin{array}{l}\text { Ref. } \\
0.99 \\
(0.68-1.46)\end{array}$ & $\begin{array}{l}11 \\
29\end{array}$ & $\begin{array}{l}\text { Ref. } \\
0.66 \\
(0.32-1.37)\end{array}$ & $\begin{array}{r}58 \\
196\end{array}$ & $\begin{array}{l}\text { Ref. } \\
0.84 \\
(0.59-1.20)\end{array}$ \\
\hline $\begin{array}{l}\text { DNMT3b C } \rightarrow T \\
(\text { rs2424913) }\end{array}$ & $\begin{array}{l}C C \\
C T+T T\end{array}$ & $\begin{array}{l}23 \\
57\end{array}$ & $\begin{array}{l}\text { Ref. } \\
1.24 \\
(0.74-2.06)\end{array}$ & $\begin{array}{r}66 \\
121\end{array}$ & $\begin{array}{l}\text { Ref. } \\
0.91 \\
(0.65-1.27)\end{array}$ & $\begin{array}{l}23 \\
55\end{array}$ & $\begin{array}{l}\text { Ref. } \\
1.18 \\
(0.71-1.97)\end{array}$ & $\begin{array}{r}80 \\
141\end{array}$ & $\begin{array}{l}\text { Ref. } \\
0.88 \\
(0.64-1.21)\end{array}$ & $\begin{array}{l}10 \\
31\end{array}$ & Ref. & $\begin{array}{r}92 \\
165\end{array}$ & $\begin{array}{l}\text { Ref. } \\
0.89 \\
(0.66-1.21)\end{array}$ \\
\hline $\begin{array}{l}\text { DNMT3b C } \rightarrow T \\
\text { (rs406193) }\end{array}$ & $\begin{array}{l}C C \\
C T+T T\end{array}$ & $\begin{array}{l}61 \\
19\end{array}$ & $\begin{array}{l}\text { Ref. } \\
0.74 \\
(0.43-1.29)\end{array}$ & $\begin{array}{r}145 \\
40\end{array}$ & $\begin{array}{l}\text { Ref. } \\
0.67 \\
(0.45-0.98)\end{array}$ & $\begin{array}{l}60 \\
17\end{array}$ & $\begin{array}{l}\text { Ref. } \\
0.69 \\
(0.39-1.21)\end{array}$ & $\begin{array}{r}167 \\
51\end{array}$ & $\begin{array}{l}\text { Ref. } \\
0.73 \\
(0.51-1.05)\end{array}$ & $\begin{array}{l}30 \\
10\end{array}$ & Ref. & $\begin{array}{r}196 \\
57\end{array}$ & $\begin{array}{l}\text { Ref. } \\
0.70 \\
(0.50-0.98)\end{array}$ \\
\hline $\begin{array}{l}\text { EHMT1 G } \rightarrow A \\
(\mathrm{rs} 4634736)\end{array}$ & $\begin{array}{l}G G \\
G A+A A\end{array}$ & $\begin{array}{l}69 \\
11\end{array}$ & $\begin{array}{l}\text { Ref. } \\
0.62 \\
(0.32-1.21)\end{array}$ & $\begin{array}{r}142 \\
43\end{array}$ & $\begin{array}{l}\text { Ref. } \\
1.19 \\
(0.81-1.75)\end{array}$ & $\begin{array}{l}62 \\
15\end{array}$ & $\begin{array}{l}\text { Ref. } \\
1.01 \\
(0.56-1.82)\end{array}$ & $\begin{array}{r}175 \\
44\end{array}$ & $\begin{array}{l}\text { Ref. } \\
0.98 \\
(0.67-1.44)\end{array}$ & $\begin{array}{r}37 \\
3\end{array}$ & Ref. & $\begin{array}{r}199 \\
56\end{array}$ & $\begin{array}{l}\text { Ref. } \\
1.10 \\
(0.78-1.57)\end{array}$ \\
\hline $\begin{array}{l}\text { EHMT2 G } \rightarrow A \\
\text { (rs535586) }\end{array}$ & $\begin{array}{l}G G \\
G A+A A\end{array}$ & $\begin{array}{l}35 \\
44\end{array}$ & $\begin{array}{l}\text { Ref. } \\
0.82 \\
(0.51-1.32)\end{array}$ & $\begin{array}{r}86 \\
143\end{array}$ & $\begin{array}{l}\text { Ref. } \\
0.75 \\
(0.54-1.04)\end{array}$ & $\begin{array}{l}35 \\
42\end{array}$ & $\begin{array}{l}\text { Ref. } \\
0.80 \\
(0.50-1.28)\end{array}$ & $\begin{array}{l}102 \\
114\end{array}$ & $\begin{array}{l}\text { Ref. } \\
0.73 \\
(0.54-1.00)\end{array}$ & $\begin{array}{l}15 \\
25\end{array}$ & $\begin{array}{l}\text { Ref. } \\
1.07 \\
(0.56-2.07)\end{array}$ & $\begin{array}{l}119 \\
132\end{array}$ & $\begin{array}{l}\text { Ref. } \\
0.73 \\
(0.54-0.98)\end{array}$ \\
\hline $\begin{array}{l}P R D M 2 G \rightarrow A \\
(\mathrm{rs} 2235515)\end{array}$ & $\begin{array}{l}G G \\
G A+A A\end{array}$ & $\begin{array}{l}49 \\
31\end{array}$ & $\begin{array}{l}\text { Ref. } \\
0.94 \\
(0.58-1.51)\end{array}$ & $\begin{array}{r}99 \\
109\end{array}$ & $\begin{array}{l}\text { Ref. } \\
1.22 \\
(0.87-1.69)\end{array}$ & $\begin{array}{l}39 \\
37\end{array}$ & $\begin{array}{l}\text { Ref. } \\
1.41 \\
(0.88-2.26)\end{array}$ & $\begin{array}{r}122 \\
91\end{array}$ & $\begin{array}{l}\text { Ref. } \\
1.11 \\
(0.81-1.51)\end{array}$ & $\begin{array}{l}21 \\
20\end{array}$ & $\begin{array}{l}\text { Ref. } \\
1.41 \\
(0.75-2.66)\end{array}$ & $\begin{array}{l}137 \\
111\end{array}$ & $\begin{array}{l}\text { Ref. } \\
1.20 \\
(0.90-1.61)\end{array}$ \\
\hline
\end{tabular}

*Number of cases; subgroups of $\leq 10$ cases were considered too small for precise estimates and were therefore omitted from the table.

${ }^{+}$Age-adjusted incidence rate ratios $(95 \% \mathrm{CI})$.

$\ddagger_{\text {dbSNP number. }}$

Netherlands. We observed that MTHFR C677T may have opposite effects in men and women and that MTR A2756G potentially increases colorectal cancer risk. In addition, genetic variants of DNMT3b and EHMT2 may reduce colorectal cancer risk among women. Rare variants of MTR and MTRR were inversely associated with CIMP in men and MLH1 hypermethylation in women, respectively, possibly by lowering methyl group availability. Although methylation-associated characteristics were significantly related, the overlap between CIMP and MLH1 hypermethylation or microsatellite instability was limited.

The MTHFR C677T and A1298C polymorphisms have previously been investigated. Rare variants of these polymorphisms were generally inversely associated with colorectal cancer risk $(4,5,12,44,45)$ or with colorectal adenoma recurrence (46). Conversely, associations with increased colorectal cancer risk (47-49), colorectal adenoma risk (50), or increased risk for colorectal adenoma recurrence $(51,52)$ have been observed as well. Analyses were stratified by sex in some studies, and positive associations with colorectal cancer were reported among men for MTHFR C677T (47) and A1298C (48, 50). In addition, similar to our study, the rare $T$ allele of the MTHFR C677T polymorphism has been observed to be associated with increased colorectal cancer risk among women but not among men (48). MTHFR polymorphisms may reduce enzymatic activity and thereby decrease the pool of 5-methyltetrahydrofolate. The resulting lower availability of methyl groups may lead to global DNA hypomethylation (53). It may also be associated with increased promoter hypermethylation in colorectal cancer (7), with CIMP (6), or with microsatellite instability $(8,54$, $55)$, which is highly correlated with CIMP in colorectal cancer (36). Although these two opposite methylation patterns may develop independently of one another, global DNA hypomethylation and gene promoter hypermethylation are observed concurrently in colorectal cancer (56-58). However, it is currently unknown to what extent alterations in methyl group metabolism influence this imbalance in the distribution of cytosine methylation, and neither do we know to what extent this influences colorectal carcinogenesis. Moreover, methyl groups may have different targets, for example, DNA, RNA, or proteins (Fig. 1), and the relative contribution of methyl groups to either of these targets is unknown.

Although, in our study, MTHFR C677T tended to increase overall colorectal cancer risk among women, this effect could not be explained by a distinct positive association with CIMP only. Among men on the other hand, the MTHFR 677TT genotype was associated with reduced overall colorectal cancer risk, and the $C$ allele of MTHFR $A 1298 C$ was inversely associated with colorectal cancers without CIMP. An increased pool of 5,10-methylenene tetrahydrofolate by MTHFR polymorphisms may optimize DNA synthesis and lower the incorporation of uracil into DNA (59). However, this may have dual consequences for carcinogenesis because increased synthesis and stability of DNA possibly protects against carcinogenesis but may also increase cell proliferation in tumors. Nonetheless, we hypothesize that MTHFR may act as a switch being able to shift the balance between DNA methylation 
and DNA synthesis (Fig. 1), both of which may have distinct consequences for carcinogenesis. Our observations suggest that MTHFR polymorphisms, albeit in different directions, may influence colorectal cancer risk in men and women but that a change in promoter hypermethylation, as measured by CIMP or MLH1 hypermethylation, may not be the primary contributor to carcinogenesis in these individuals.

Among subjects in our study population, the MTR 2756GG genotype was associated with increased colorectal cancer risk. Genetic variants of MTR were not associated with colorectal cancer in a number of previous studies $(12,60,61)$. Modest risk reductions among MTR 2756GG homozygotes were found in one colorectal adenoma study (9) and in one colorectal cancer study (10), whereas also nonsignificant increased risks were observed between this single-nucleotide polymorphism and colorectal adenoma (11) or colorectal cancer (13). Rare variants of MTR were associated with lower plasma homocysteine concentrations (62), suggesting lower catalytic activity of the MTR enzyme and lower availability of methyl groups. Interestingly, we observed that the MTR A2756G single-nucleotide polymorphism was associated with reduced risk for colorectal cancers with CIMP among men.

It was previously observed that rare variants of the MTRR A66G single-nucleotide polymorphism may be inversely associated with colorectal adenoma recurrence (46) but with increased colorectal cancer risk (13). In addition, other single-nucleotide polymorphisms in MTRR were associated with an increased risk for colorectal adenoma (11) and colorectal cancer (12). We did not observe an association with overall colorectal cancer risk in our study, but the $G$ allele of the MTRR A66G polymorphism was associated with reduced risk for colorectal cancers with MLH1 hypermethylation among women. The MTRR A66G single-nucleotide polymorphism may also be associated with reduced catalytic activity of the MTRR enzyme (63). Our data suggest that this possibly results in decreased promoter hypermethylation in colorectal cancer similar to MTR A2756G, which is involved in the same metabolic conversion in the one-carbon metabolism.

The CIMP phenotype has not uniquely been defined because various sets of promoters have previously been used with different cutoff values (64). To investigate an optimal set of markers for CIMP, Weisenberger et al. (36) screened 195 CpG island methylation markers and proposed a robust new panel of five markers to define CIMP in colorectal cancer. This new set has been validated successfully in a large group of incident colorectal cancer patients (65), and we have used these new markers in our study. However, we have shown that the overlap between CIMP and MLH1 or microsatellite instability may be incomplete. It has also been suggested that CIMP may consist of three molecular subtypes based on presence or absence of microsatellite instability, BRAF, KRAS, or p53 mutations (66), but these characteristics are not mutually exclusive. In addition, we previously observed incomplete overlap between MLH1 hypermethylation or expression, microsatellite instability, and $B R A F$ mutations (41). The current study suggests that lower enzymatic activity of folate metabolizing enzymes MTR and MTRR is inversely associated with either CIMP or MLH1 hypermethylation. In view of these observations, it remains important to investigate associations with CIMP and other methylation endpoints separately.
The DNMT3b $C \rightarrow T$ (rs2424913) single-nucleotide polymorphism was not associated with colorectal cancer risk. However, an increased risk for colorectal cancer could be expected because this polymorphism increases enzymatic activity of DNMT3b (21), and DNMT3b overexpression was associated with increased promoter hypermethylation in different types of cancers $(15-17,19,20)$. Moreover, positive associations were observed with colorectal polyps and early onset of colorectal cancer in hereditary nonpolyposis colorectal cancer patients $(23,25)$ and several other cancers $(21,22,24)$. Other genetic variants of $D N M T 3 b$ that have been identified are the $-283 T \rightarrow C$ and $-579 G \rightarrow T$ polymorphisms, of which the latter was associated with reduced colorectal cancer risk (67). In addition, lung cancer risk was reduced in individuals carrying rare alleles of each of these single-nucleotide polymorphisms (68), which was contrary to the increased lung cancer risk associated with $D N M T 3 b C \rightarrow T$ (rs2424913) observed by Shen et al. (21). Interestingly, enzymatic activity of DNMT3b was decreased when the rare allele of $-283 T \rightarrow C$ was present (68). Apparently, different polymorphisms in DNMT3b may have opposite effects and individuals may be more susceptible to carcinogenesis with higher DNMT3b enzymatic activity $(21-23,25)$, whereas decreased activity may protect against carcinogenesis (67, 68). Whereas a similar but weak inverse association was previously observed with breast cancer for the DNMT3b $C \rightarrow T$ (rs406193) polymorphism (30), we observed an inverse association with colorectal cancer among women. However, this protective effect was not substantially different between colorectal cancers with or without CIMP, MLH1 hypermethylation, or microsatellite instability.

For histone methyltransferases, we observed that the rare A allele of $E H M T 2 G \rightarrow A$ (rs535586) was inversely associated with colorectal cancer among women. A significant positive association was observed with breast cancer, although the incidence rate ratio was small for that particular polymorphism (30). These observations seem contradictive but may nonetheless suggest that this EHMT2 polymorphism affects carcinogenesis of different cancer types. Importantly, it is currently unknown to which extent single-nucleotide polymorphisms in histone methyltransferases such as EHMT2 lead to altered enzymatic activity. Obviously, the exact role of EHMT2 in colorectal carcinogenesis needs further investigation.

This is the first prospective cohort study reporting associations between genetic variants of DNA methyltransferases and histone methyltransferases with colorectal cancer risk, suggesting the importance of such epigenetic regulators in the carcinogenesis of colorectal cancer. The analyses were stratified for gender and molecular phenotypes of the tumor. Therefore, several tests were done for each single-nucleotide polymorphism, which may have increased the danger of reporting chance findings. It could also be argued that some of these subgroups should preferably have been larger. For these reasons, caution must be taken in drawing definite conclusions, and the findings should be replicated in future research.

In conclusion, genetic variants of methyl metabolism enzymes and/or epigenetic regulators may affect colorectal carcinogenesis, and our observations suggest that reduced enzymatic activity of some folate metabolizing enzymes may result in decreased gene promoter hypermethylation in colorectal cancer. Hence, it would 
be interesting to study whether methyl donor intake affects promoter hypermethylation and the potential modifying effect of polymorphisms in folate metabolizing enzymes, DNA methyltransferases, and histone methyltransferases. We observed that the overlap of CpG island hypermethylation markers and associated characteristics may be incomplete, indicating that these characteristics should be considered separately when studying potential causes of a methylation phenotype in colorectal cancer.

\section{Disclosure of Potential Conflicts of Interest}

No potential conflicts of interest were disclosed.

\section{Acknowledgments}

The costs of publication of this article were defrayed in part by the payment of page charges. This article must therefore be hereby marked advertisement in accordance with 18 U.S.C. Section 1734 solely to indicate this fact.

We thank Dr. M. Brink for the collection of the tissue samples.

\section{References}

1. Herman JG, Baylin SB. Gene silencing in cancer in association with promoter hypermethylation. N Engl J Med 2003;349:2042-54.

2. Frosst P, Blom HJ, Milos R, et al. A candidate genetic risk factor for vascular disease: a common mutation in methylenetetrahydrofolate reductase. Nat Genet 1995;10:111-3.

3. van der Put NM, Gabreels F, Stevens EM, et al. A second common mutation in the methylenetetrahydrofolate reductase gene: an additional risk factor for neural-tube defects? Am J Hum Genet 1998;62: 1044-51.

4. Huang Y, Han S, Li Y, Mao Y, Xie Y. Different roles of MTHFR C677T and A1298C polymorphisms in colorectal adenoma and colorectal cancer: a meta-analysis. J Hum Genet 2007;52:73-85.

5. Hubner RA, Houlston RS. MTHFR C677T and colorectal cancer risk: a meta-analysis of 25 populations. Int J Cancer 2007;120:1027-35.

6. Curtin K, Slattery ML, Ulrich CM, et al. Genetic polymorphisms in one-carbon metabolism: associations with CPG island methylator phenotype (CIMP) in colon cancer and the modifying effects of diet. Carcinogenesis 2007;28:1672-9.

7. Oyama K, Kawakami K, Maeda K, Ishiguro K, Watanabe G. The association between methylenetetrahydrofolate reductase polymorphism and promoter methylation in proximal colon cancer. Anticancer Res 2004;24:649-54.

8. Hubner RA, Lubbe S, Chandler I, Houlston RS. MTHFR C677T has differential influence on risk of MSI and MSS colorectal cancer. Hum Mol Genet 2007; 16:1072-7.

9. Chen J, Giovannucci E, Hankinson SE, et al. A prospective study of methylenetetrahydrofolate reductase and methionine synthase gene polymorphisms, and risk of colorectal adenoma. Carcinogenesis 1998;19:2129-32.

10. Ma J, Stampfer MJ, Christensen B, et al. A polymorphism of the methionine synthase gene: association with plasma folate, vitamin B12, homocyst(e)ine, and colorectal cancer risk. Cancer Epidemiol Biomarkers Prev 1999;8:825-9.

11. Hazra A, Wu K, Kraft P, Fuchs CS, Giovannucci EL, Hunter DJ. Twentyfour non-synonymous polymorphisms in the one-carbon metabolic pathway and risk of colorectal adenoma in the Nurses' Health Study. Carcinogenesis 2007;28:1510-9.

12. Koushik A, Kraft P, Fuchs CS, et al. Nonsynonymous polymorphisms in genes in the one-carbon metabolism pathway and associations with colorectal cancer. Cancer Epidemiol Biomarkers Prev 2006;15:2408-17.

13. Le Marchand L, Donlon T, Hankin JH, Kolonel LN, Wilkens LR, Seifried A. B-vitamin intake, metabolic genes, and colorectal cancer risk (United States). Cancer Causes Control 2002;13:239-48.

14. Okano M, Bell DW, Haber DA, Li E. DNA methyltransferases Dnmt3a and Dnmt3b are essential for de novo methylation and mammalian development. Cell 1999;99:247-57.

15. Beaulieu N, Morin S, Chute IC, Robert MF, Nguyen H, MacLeod AR. An essential role for DNA methyltransferase DNMT3B in cancer cell survival. J Biol Chem 2002;277:28176-81.

16. Jacinto FV, Ballestar E, Ropero S, Esteller M. Discovery of epigenetically silenced genes by methylated DNA immunoprecipitation in colon cancer cells. Cancer Res 2007;67:11481-6.
17. Wang J, Bhutani M, Pathak AK, et al. $\delta$ DNMT3B variants regulate DNA methylation in a promoter-specific manner. Cancer Res 2007;67: 10647-52.

18. Linhart HG, Lin H, Yamada $Y$, et al. Dnmt $3 b$ promotes tumorigenesis in vivo by gene-specific de novo methylation and transcriptional silencing. Genes Dev 2007;21:3110-22.

19. Wang J, Walsh G, Liu DD, Lee JJ, Mao L. Expression of $\delta$ DNMT3B variants and its association with promoter methylation of p16 and RASSF1A in primary non-small cell lung cancer. Cancer Res 2006;66: 8361-6.

20. Roll JD, Rivenbark AG, Jones WD, Coleman WB. DNMT3b overexpression contributes to a hypermethylator phenotype in human breast cancer cell lines. Mol Cancer 2008;7:15.

21. Shen H, Wang L, Spitz MR, Hong WK, Mao L, Wei Q. A novel polymorphism in human cytosine DNA-methyltransferase-3B promoter is associated with an increased risk of lung cancer. Cancer Res 2002;62: 4992-5.

22. Singal R, Das PM, Manoharan M, Reis IM, Schlesselman JJ. Polymorphisms in the DNA methyltransferase $3 \mathrm{~b}$ gene and prostate cancer risk. Oncol Rep 2005;14:569-73.

23. Jung AY, Poole EM, Bigler J, Whitton J, Potter JD, Ulrich CM. DNA methyltransferase and alcohol dehydrogenase: gene-nutrient interactions in relation to risk of colorectal polyps. Cancer Epidemiol Biomarkers Prev 2008;17:330-8.

24. Wang L, Rodriguez M, Kim ES, et al. A novel C/T polymorphism in the core promoter of human de novo cytosine DNA methyltransferase 3B6 is associated with prognosis in head and neck cancer. Int J Oncol 2004;25:993-9.

25. Jones JS, Amos CI, Pande M, et al. DNMT3b polymorphism and hereditary nonpolyposis colorectal cancer age of onset. Cancer Epidemiol Biomarkers Prev 2006;15:886-91.

26. Tamaru H, Selker EU. A histone $\mathrm{H} 3$ methyltransferase controls DNA methylation in Neurospora crassa. Nature 2001;414:277-83.

27. Kondo Y, Shen L, Issa JP. Critical role of histone methylation in tumor suppressor gene silencing in colorectal cancer. Mol Cell Biol 2003;23: 206-15.

28. Chadwick RB, Jiang GL, Bennington GA, et al. Candidate tumor suppressor RIZ is frequently involved in colorectal carcinogenesis. Proc Natl Acad Sci U S A 2000;97:2662-7.

29. Yoon KA, Park S, Hwangbo B, et al. Genetic polymorphisms in the $\mathrm{Rb}$-binding zinc finger gene RIZ and the risk of lung cancer. Carcinogenesis 2007;28:1971-7.

30. Cebrian A, Pharoah PD, Ahmed S, et al. Genetic variants in epigenetic genes and breast cancer risk. Carcinogenesis 2006;27:1661-9.

31. van den Brandt PA, Goldbohm RA, van 't Veer $P$, Volovics A, Hermus RJ, Sturmans F. A large-scale prospective cohort study on diet and cancer in The Netherlands. J Clin Epidemiol 1990;43:285-95.

32. Van den Brandt PA, Schouten LJ, Goldbohm RA, Dorant E, Hunen PM. Development of a record linkage protocol for use in the Dutch Cancer Registry for Epidemiological Research. Int J Epidemiol 1990; 19:553-8.

33. Casparie M, Tiebosch AT, Burger G, et al. Pathology databanking and biobanking in The Netherlands, a central role for PALGA, the nationwide histopathology and cytopathology data network and archive. Cell Oncol 2007;29:19-24.

34. Meulenbelt I, Droog S, Trommelen GJ, Boomsma DI, Slagboom PE. High-yield noninvasive human genomic DNA isolation method for genetic studies in geographically dispersed families and populations. Am J Hum Genet 1995;57:1252-4.

35. Knaapen AM, Ketelslegers HB, Gottschalk RW, et al. Simultaneous genotyping of nine polymorphisms in xenobiotic-metabolizing enzymes by multiplex PCR amplification and single base extension. Clin Chem 2004;50:1664-8.

36. Weisenberger DJ, Siegmund KD, Campan M, et al. CpG island methylator phenotype underlies sporadic microsatellite instability and is tightly associated with BRAF mutation in colorectal cancer. Nat Genet 2006;38:787-93.

37. Herman JG, Graff JR, Myohanen S, Nelkin BD, Baylin SB. Methylation-specific PCR: a novel PCR assay for methylation status of CpG islands. Proc Natl Acad Sci U S A 1996;93:9821-6.

38. van Engeland M, Weijenberg MP, Roemen GM, et al. Effects of dietary folate and alcohol intake on promoter methylation in sporadic colorectal cancer: the Netherlands cohort study on diet and cancer. Cancer Res 2003;63:3133-7.

39. Derks S, Lentjes MH, Hellebrekers DM, de Bruine AP, Herman JG, van Engeland M. Methylation-specific PCR unraveled. Cell Oncol 2004;26: 291-9.

40. Suraweera N, Duval A, Reperant M, et al. Evaluation of tumor microsatellite instability using five quasimonomorphic mononucleotide repeats and pentaplex PCR. Gastroenterology 2002;123:1804-11.

41. de Vogel S, Bongaerts BW, Wouters KA, et al. Associations of dietary 
methyl donor intake with MLH1 promoter hypermethylation and related molecular phenotypes in sporadic colorectal cancer. Carcinogenesis 2008;29:1765-73.

42. Lin DYWL. The robust inference for the Cox proportional hazards model. J Amer Statistical Assoc 1989;84:1074-8.

43. Schoenfeld D. Partial residuals for the proportional hazards regression models. Biometrika 1982;69:239-41.

44. Sharp L, Little J, Brockton NT, et al. Polymorphisms in the methylenetetrahydrofolate reductase (MTHFR) gene, intakes of folate and related B vitamins and colorectal cancer: a case-control study in a population with relatively low folate intake. Br J Nutr 2008;99:379-89.

45. Murtaugh MA, Curtin K, Sweeney C, et al. Dietary intake of folate and co-factors in folate metabolism, MTHFR polymorphisms, and reduced rectal cancer. Cancer Causes Control 2007;18:153-63.

46. Hubner RA, Muir KR, Liu JF, et al. Folate metabolism polymorphisms influence risk of colorectal adenoma recurrence. Cancer Epidemiol Biomarkers Prev 2006;15:1607-13.

47. Heijmans BT, Boer JM, Suchiman HE, et al. A common variant of the methylenetetrahydrofolate reductase gene (1p36) is associated with an increased risk of cancer. Cancer Res 2003;63:1249-53.

48. Lightfoot TJ, Barrett JH, Bishop T, et al. Methylene tetrahydrofolate reductase genotype modifies the chemopreventive effect of folate in colorectal adenoma, but not colorectal cancer. Cancer Epidemiol Biomarkers Prev 2008;17:2421-30.

49. Ryan BM, Molloy AM, McManus R, et al. The methylenetetrahydrofolate reductase (MTHFR) gene in colorectal cancer: role in tumor development and significance of allelic loss in tumor progression. Int $\mathrm{J}$ Gastrointest Cancer 2001;30:105-11.

50. Mitrou PN, Watson MA, Loktionov AS, et al. MTHFR (C677T and A1298C) polymorphisms and risk of sporadic distal colorectal adenoma in the UK Flexible Sigmoidoscopy Screening Trial (United Kingdom). Cancer Causes Control 2006;17:793-801.

51. Martinez ME, Thompson P, Jacobs ET, et al. Dietary factors and biomarkers involved in the methylenetetrahydrofolate reductase genotype-colorectal adenoma pathway. Gastroenterology 2006;131: 1706-16.

52. Murphy G, Sansbury LB, Cross AJ, et al. Folate and MTHFR: risk of adenoma recurrence in the Polyp Prevention Trial. Cancer Causes Control 2008;19:751-8.

53. Friso S, Choi SW, Girelli D, et al. A common mutation in the 5,10methylenetetrahydrofolate reductase gene affects genomic DNA methylation through an interaction with folate status. Proc Natl Acad Sci U S A 2002;99:5606-11.

54. Chang SC, Lin PC, Lin JK, Yang SH, Wang HS, Fen-Yau Li A. Role of MTHFR polymorphisms and folate levels in different phenotypes of sporadic colorectal cancers. Int J Colorectal Dis 2007;22:483-9.
55. Clarizia AD, Bastos-Rodrigues L, Pena HB, et al. Relationship of the methylenetetrahydrofolate reductase C677T polymorphism with microsatellite instability and promoter hypermethylation in sporadic colorectal cancer. Genet Mol Res 2006;5:315-22.

56. Ehrlich M. Cancer-linked DNA hypomethylation and its relationship to hypermethylation. Curr Top Microbiol Immunol 2006;310:251-74.

57. Bariol C, Suter C, Cheong K, et al. The relationship between hypomethylation and $\mathrm{CpG}$ island methylation in colorectal neoplasia. Am J Pathol 2003;162:1361-71.

58. Frigola J, Sole X, Paz MF, et al. Differential DNA hypermethylation and hypomethylation signatures in colorectal cancer. Hum Mol Genet 2005;14:319-26.

59. DeVos L, Chanson A, Liu Z, et al. Associations between single nucleotide polymorphisms in folate uptake and metabolizing genes with blood folate, homocysteine, and DNA uracil concentrations. Am J Clin Nutr 2008;88:1149-58.

60. Theodoratou E, Farrington SM, Tenesa A, et al. Dietary vitamin b6 intake and the risk of colorectal cancer. Cancer Epidemiol Biomarkers Prev 2008;17:171-82.

61. Ulrich CM, Curtin K, Potter JD, Bigler J, Caan B, Slattery ML. Polymorphisms in the reduced folate carrier, thymidylate synthase, or methionine synthase and risk of colon cancer. Cancer Epidemiol Biomarkers Prev 2005;14:2509-16.

62. Chen J, Stampfer MJ, Ma J, et al. Influence of a methionine synthase (D919G) polymorphism on plasma homocysteine and folate levels and relation to risk of myocardial infarction. Atherosclerosis 2001; 154:667-72.

63. Gaughan DJ, Kluijtmans LA, Barbaux S, et al. The methionine synthase reductase (MTRR) A66G polymorphism is a novel genetic determinant of plasma homocysteine concentrations. Atherosclerosis 2001;157:451-6.

64. Teodoridis JM, Hardie C, Brown R. CpG island methylator phenotype (CIMP) in cancer: causes and implications. Cancer Lett 2008; 268:177-86.

65. Ogino S, Kawasaki T, Kirkner GJ, Kraft P, Loda M, Fuchs CS. Evaluation of markers for $\mathrm{CpG}$ island methylator phenotype (CIMP) in colorectal cancer by a large population-based sample. J Mol Diagn 2007; 9:305-14.

66. Shen L, Toyota M, Kondo $\mathrm{Y}$, et al. Integrated genetic and epigenetic analysis identifies three different subclasses of colon cancer. Proc Natl Acad Sci U S A 2007;104:18654-9.

67. Hong YS, Lee HJ, You CH, et al. DNMT3b 39179GT polymorphism and the risk of adenocarcinoma of the colon in Koreans. Biochem Genet 2007;45:155-63.

68. Lee SJ, Jeon HS, Jang JS, et al. DNMT3B polymorphisms and risk of primary lung cancer. Carcinogenesis 2005;26:403-9. 


\section{Cancer Epidemiology, Biomarkers \& Prevention}

\section{Genetic Variants of Methyl Metabolizing Enzymes and Epigenetic Regulators: Associations with Promoter CpG Island Hypermethylation in Colorectal Cancer}

Stefan de Vogel, Kim A.D. Wouters, Ralph W.H. Gottschalk, et al.

Cancer Epidemiol Biomarkers Prev 2009;18:3086-3096. Published OnlineFirst October 20, 2009.

\section{Updated version Access the most recent version of this article at:} doi:10.1158/1055-9965.EPI-09-0289

Supplementary Access the most recent supplemental material at:

Material http://cebp.aacrjournals.org/content/suppl/2009/10/20/1055-9965.EPI-09-0289.DC1

Cited articles This article cites 68 articles, 24 of which you can access for free at:

http://cebp.aacrjournals.org/content/18/11/3086.full\#ref-list-1

Citing articles This article has been cited by 7 HighWire-hosted articles. Access the articles at:

http://cebp.aacrjournals.org/content/18/11/3086.full\#related-urls

E-mail alerts Sign up to receive free email-alerts related to this article or journal.

Reprints and To order reprints of this article or to subscribe to the journal, contact the AACR Publications Subscriptions Department at pubs@aacr.org.

Permissions To request permission to re-use all or part of this article, use this link http://cebp.aacrjournals.org/content/18/11/3086.

Click on "Request Permissions" which will take you to the Copyright Clearance Center's (CCC)

Rightslink site. 